Document downloaded from:

http://hdl.handle.net/10251/148243

This paper must be cited as:

Gonzalez-German, IT.; Sala, A.; Bernal Reza, MA.; Robles-Ruiz, R. (2017). PiecewiseTakagi-Sugeno asymptotically exact estimation of the domain of attraction of nonlinear systems. Journal of the Franklin Institute. 354(3):1514-1541. https://doi.org/10.1016/j.jfranklin.2016.11.033

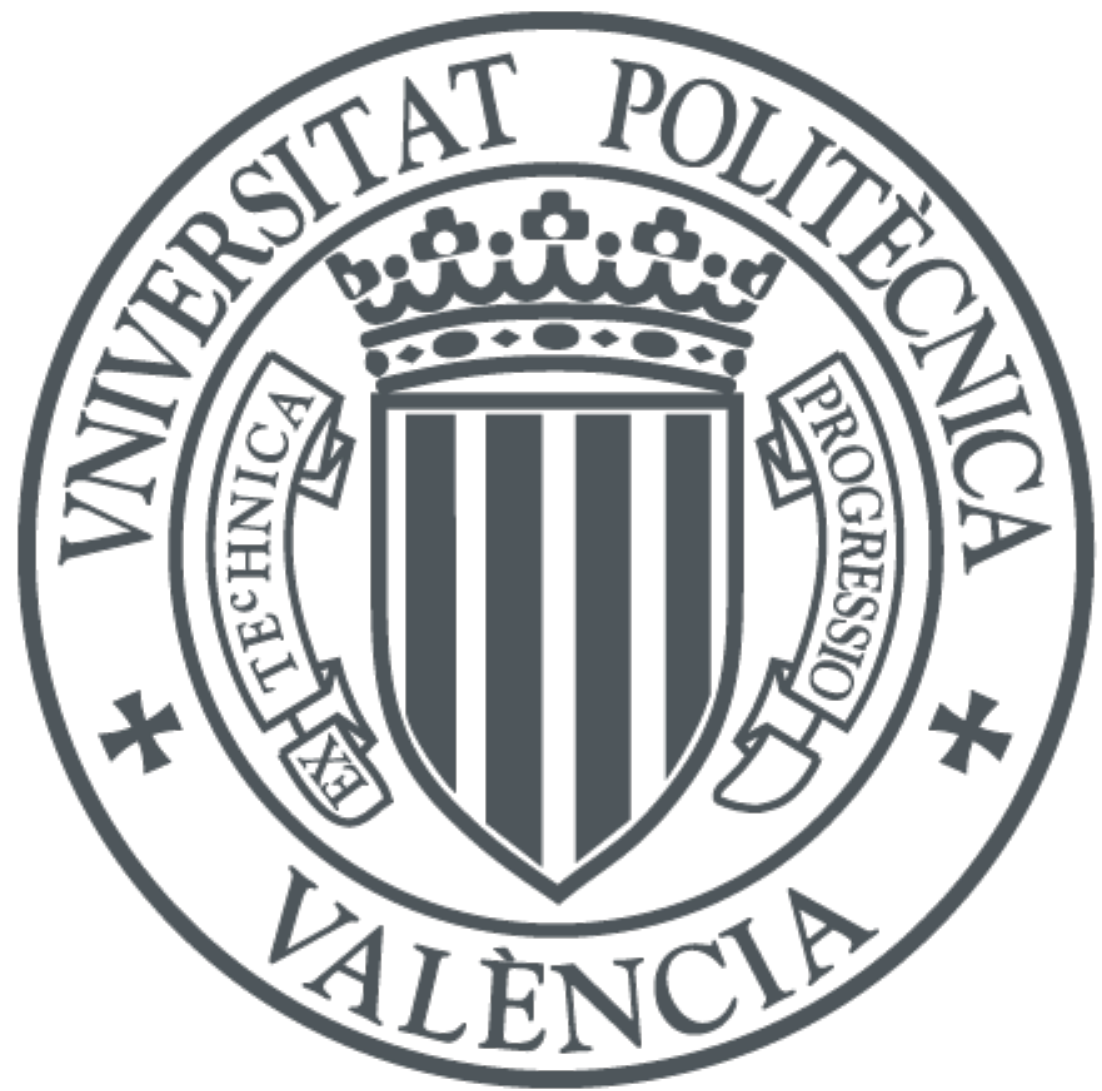

The final publication is available at

https://doi.org/10.1016/j.jfranklin.2016.11.033

Copyright Elsevier

Additional Information 


\title{
Piecewise-Takagi-Sugeno Asymptotically Exact Estimation of the Domain of Attraction of Nonlinear Systems
}

\author{
Temoatzin González ${ }^{\mathrm{a}}$, Antonio Sala ${ }^{\mathrm{a}}$, Miguel Bernal ${ }^{\mathrm{b}}$, Ruben Robles $^{\mathrm{a}}$ \\ ${ }^{a}$ Instituto Universitario de Automática e Informática Industrial, Universitat Politécnica de \\ Valencia, Camino de Vera S/N, 46022, Valencia, Spain. \\ ${ }^{b}$ Department of Electrical and Electronics Engineering, Sonora Institute of Technology, 5 de \\ Febrero 818 Sur, 85000, Ciudad Obregón, Mexico.
}

\begin{abstract}
This report generalises recent results on stability analysis and estimation of the domain of attraction of nonlinear systems via exact piecewise affine TakagiSugeno models. Algorithms in the form of linear matrix inequalities are proposed that produce progressively better estimates which are proved to asymptotically render the actual domain of attraction; regions already proven to belong to such domain of attraction can be removed and the estimate can contain significant portions of the modelling region boundary; in this way, level-set approaches in prior literature can be significantly improved. Illustrative examples and comparisons are provided.
\end{abstract}

Keywords: Domain of Attraction, Stability, Piecewise Lyapunov Function, Piecewise Affine Takagi-Sugeno, Linear Matrix Inequalities

\section{Introduction}

Takagi-Sugeno (TS) models, systematically obtained via the sector nonlinearity approach [1], have proved to be suitable for generalisation of linear techniques to handle nonlinear stability issues [2], since they are convex sums of linear systems weighted by membership functions (MFs). When combined with

\footnotetext{
* Corresponding author

Email address: temoatzin.g@gmail.com (Temoatzin González)
} 
the direct Lyapunov method, TS models naturally lead to linear matrix inequalities (LMIs) [3], which can be efficiently solved via convex optimization techniques already implemented in commercially available software [4]. The TS modelling approach has been also extended to distributed-parameter systems governed by partial differential equations $[5,6]$; nevertheless, this class of systems are out of the scope of this work.

Though the TS and nonlinear models are locally equivalent in some compact $\Omega$, also known as the modelling region, the LMI stability analysis is conservative $[7,8,9]$. This is mainly due to the fact that only vertex (linear) models are considered, i.e., MFs are ignored, thus introducing the so called shape-independent conservatism [7].

Within shape-independent approaches, piecewise analysis is known for reducing conservatism by lowering the separation among the vertex models via a partition of $\Omega$. Moreover, affine terms can be introduced in TS models if the region under consideration does not include the origin [10]. This allows considering more general piecewise-quadratic Lyapunov functions (PWQLF) [11]; other piecewise options are considered in $[12,13,14]$ for stability analysis. Piecewise TS approaches for control design have also been reported but they usually are in BMI form [15]; the work [16] presents a piecewise control synthesis procedure keeping the LMI structure, at the cost of conservatism in some steps; as we discuss a non-conservative stability-analysis setup, the issues in [16] will not be considered here. Practical applications of affine TS models appear in, for instance, [17], and those of piecewise models have been reported in [18].

The problem to be addressed in this paper is the determination of the "largest" estimate of the domain of attraction (DA) of the origin of a nonlinear system $\dot{x}=f(x)$ in a modelling region $\Omega$. To be precise, considering every conceivable $\mathscr{C}^{2}$ Lyapunov function which might exist for a system with continuous $f(\cdot)$, with enough computational resources, the proposal will prove any point in the interior of the union of all level sets (see below) in $\Omega$ to be part of the DA.

The problem of estimating the DA has been partially addressed in prior liter- 
ature. Indeed, if $0 \in \Omega$, level sets of Lyapunov functions for which $\dot{V}<-\gamma x^{T} x$, $\gamma>0$, for all $x \in \Omega, x \neq 0$, belong to the DA; this is the approach pursued in most stability analysis proposals in literature [19]; these level sets are usually "tangent" to the boundary of $\Omega$ and have been already extended to the piecewise case [20]. However, the DA can contain significant portions of the boundary of $\Omega$ if the trajectories "point" towards its interior; hence, standard level-set results can be expanded [21]. Also, a related approach was pursued in [22] in the polynomial-fuzzy arena, introducing the idea of getting progressively better estimates of the domain of attraction by subtracting already-proven estimates. More recently, with non-piecewise models but piecewise Lyapunov functions, a shape-independent approach for maximal DA computation for TS systems has been presented in [12]; in $[15,14]$ a piecewise Lyapunov function defined by the minimum or maximum of quadratics (or higher-order polynomials) is considered. However, in such cases the delimitation of the regions is not fixed a priori and the problem ends up being a bilinear matrix inequality (BMI).

The most related prior-literature work on the ideas here is [20], based on exact piecewise affine TS models (PWATS) and iteratively changing the modelling region $\Omega$. The work here presented generalises [20], by considering the fact that level sets can exit $\Omega$, introducing more general multipliers, exploiting previously proven DA estimates (lifting decrescence and continuity constraints inside them), and modifying the above-mentioned iterations on the modelling region shape accounting for the more powerful results, within an LMI framework. The proposal in this investigation, based on the Farkas lemma, is asymptotically exact; hence, if a particular point belongs to the interior of the "true" DA, a suitable fine enough partition will prove it to belong to the DA.

This work is organized as follows: extensive preliminaries are introduced in section 2, covering the definition of DA, the different TS piecewise modelling options, basic results on piecewise stability, and the relevance of the Positivstellensatz (S-procedure) argumentation; in section 3 new results and algorithms are inferred that generalise previous approaches for estimation of the DA; the important subject of asymptotic exactness of the proposed results is treated in 
section 4; illustrative examples are given along the contents of the paper. Conclusions in section 5 gather some final remarks, and an appendix collects the proofs of the main results.

\section{Preliminaries}

Consider an autonomous nonlinear model

$$
\dot{x}(t)=f(x(t))
$$

with $x(t) \in \mathbb{R}^{n}$ as the state vector and $f(\cdot): \mathbb{R}^{n} \rightarrow \mathbb{R}^{n}$ being a $\mathscr{C}^{2}$ nonlinear vector field, i.e., with continuous second partial derivatives. By assumption, the origin will be an equilibrium point, i.e., $f(0)=0$. The solution of (1) for initial condition $x_{0}$ will be denoted as $\phi\left(t, x_{0}\right)$.

The domain of attraction [19] of $x=0$ for (1) is the set

$$
\mathcal{D}:=\left\{x \in \mathbb{R}^{n}: \lim _{t \rightarrow \infty} \phi(t, x)=0\right\}
$$

\subsection{Affine Fuzzy Modelling}

The well-known sector nonlinearity technique [1] allows finding an equivalent Takagi-Sugeno model in a compact set $\Omega$ of the state space including the origin. This work considers regions which do not contain the origin; the sectornonlinearity ideas can be generalised to such a case, following [10].

Indeed, as $f$ is linearisable at the origin, denoting as $A$ its Jacobian, we can rewrite $f$ in (1) as

$$
f(x)=A x+\sum_{j=1}^{p} M_{j} \rho_{j}(x)
$$

with $\rho_{j}: \mathbb{R}^{n} \mapsto \mathbb{R}$, for $j=\{1,2, \ldots, p\}$, being some nonlinearities whose linearisation is zero ${ }^{1}$, and $M_{j}$ being column vectors indicating how nonlinearity $\rho_{j}$

\footnotetext{
${ }^{1}$ There is no loss of generality, as the Jacobian (first-derivatives) can be embeeded in $A$; for instance, $\sin (x)=x+g(x)$, with $g(x)=\sin (x)-x, \partial g / \partial x=0$.
} 
enters in each of the equations of (1). As $\Omega$ is compact and $f$ is $\mathscr{C}^{2}$, each $\rho_{j}$ can be bounded in $\Omega$ by two affine functions:

$$
\underline{z}_{j}(x) \leq \rho_{j}(x) \leq \bar{z}_{j}(x)
$$

where:

$$
\bar{z}_{j}(x)=a_{1}^{j} H_{j} x+b_{1}^{j}, \quad \underline{z}_{j}(x)=a_{0}^{j} H_{j} x+b_{0}^{j},
$$

being $a_{i}^{j}, b_{i}^{j}$ scalars, and $H_{j}$ row vectors, configuring arbitrarily tight linear bounds on $\rho_{j}(x)$. Once the bound (4) is available, we can express:

$$
\rho_{j}(x)=\sum_{i=0}^{1} w_{i}^{j}(x)\left(a_{i}^{j} H_{j} x+b_{i}^{j}\right)
$$

being the memberships given by the well-known interpolation expression:

$$
w_{0}^{j}(x):=\frac{\bar{z}_{j}(x)-\rho_{j}(x)}{\bar{z}_{j}(x)-\underline{z}_{j}(x)}, \quad w_{1}^{j}(\cdot):=1-w_{0}^{j}(\cdot) .
$$

Operating with all $\rho_{j}$, for $j \in\{1,2, \ldots, p\}$, then $r=2^{p}$ membership functions can be defined as

$$
h_{i}(x):=\prod_{j=1}^{p} w_{i_{j}}^{j}(x),
$$

with $i \in\{1,2, \ldots, r\}$, building a binary-digit expression of $i$ as $i=i_{p} \times 2^{p-1}+$ $\ldots+i_{2} \times 2+i_{1}+1, i_{j} \in\{0,1\}$. Obviously, the MFs hold the convex sum property, i.e., $\sum_{i=1}^{r} h_{i}(x)=1, h_{i}(x) \geq 0$. Using such memberships, (1) can be expressed as:

$$
\dot{x}:=\sum_{i=1}^{r} h_{i}(x)\left(A x+\sum_{j=1}^{p} M_{j}\left(a_{i_{j}}^{j} H_{j} x+b_{i_{j}}^{j}\right)\right)
$$

If the standard shorthand notation $\Upsilon_{\mathbf{h}}:=\sum_{i=1}^{r} h_{i}(z(t)) \Upsilon_{i}$ is adopted, from (9), denoting $A_{i}:=A+\sum_{j=1}^{p} M_{j} a_{i_{j}}^{j} H_{j}$ and $b_{i}:=\sum_{j=1}^{p} M_{j} b_{i_{j}}^{j}$, the nonlinear model (1) in $\Omega$ can be compactly written as the following affine-TS model:

$$
\dot{x}(t)=A_{\mathbf{h}} x(t)+b_{\mathbf{h}}, \quad x(t) \in \Omega,
$$

Remark 1. Several options for affine piecewise TS modelling are available; the examples worked out in this paper used the minimum-weighted area approach in [10]. 


\subsubsection{Piecewise Affine TS models}

Consider a connected modelling region $\Omega$, which is partitioned into $q$ subregions with disjoint interiors, $\Omega_{k}, k \in\{1,2, \ldots, q\}$, i.e.,

$$
\bigcup_{k=1}^{q} \Omega_{k}=\Omega, \quad \operatorname{int}\left(\Omega_{k}\right) \cap \operatorname{int}\left(\Omega_{l}\right)=\emptyset .
$$

If the above-discussed affine fuzzy modelling techniques are used, we can express the original nonlinear dynamics as a piecewise affine TS model (PWATS) [11] in the form ${ }^{2}$ :

$$
\begin{gathered}
\dot{x}(t)=A_{\mathbf{h}}^{k} x(t), \quad x(t) \in \Omega_{k}, \quad k \in K_{0}, \\
\dot{x}(t)=A_{\mathbf{h}}^{k} x(t)+b_{\mathbf{h}}^{k}, \quad x(t) \in \Omega_{k}, \quad k \in K_{1},
\end{gathered}
$$

where $K_{0}:=\left\{k: 0 \in \Omega_{k}\right\}$ is the set of indexes of those regions $\Omega_{k}$ that include the origin and $K_{1}:=\left\{k: 0 \notin \Omega_{k}\right\}$ is the set of indexes of the remaining ones (not containing the origin).

For later analysis, each of the regions $\Omega_{k}$ will be described by a set of constraints $\Omega_{k}:=\left\{\sigma_{j}^{k}(x) \geq 0, j \in\left\{1,2, \ldots, n_{k}\right\}\right\}$. If $\sigma_{j}^{k}(x)$ are affine functions of $x$, the partition of $\Omega$ is a so-called polyhedral partition; these polyhedral partitions are the ones appearing in the seminal literature [11]; non-polyhedral partitions with circular boundaries are considered in [23]. Polyhedral partitions of the state space have the form $\sigma_{k}(x):=\bar{E}_{k} \bar{x} \succeq 0$, where $\bar{E}_{k}=\left[\begin{array}{ll}E_{k} & e_{k}\end{array}\right], x \in \Omega_{k}$, $k \in\{1,2, \ldots, q\}$. A systematic procedure for their construction is described in $[11,24]$. Note that if $e_{k}=0$ the inequality $E_{k} x \succeq 0$ defines a polyhedral cone with its vertex at the origin.

For each region $\Omega_{k}$, all constraints can be joined in a vector of functions $\sigma_{k}(\cdot):=\left[\begin{array}{lll}\sigma_{1}^{k}(\cdot) & \ldots & \sigma_{n_{k}}^{k}(\cdot)\end{array}\right]^{T} ;$ thus, we could define $\Omega_{k}=\left\{x: \sigma_{k}(x) \succeq 0\right\}$, where " $\succeq 0$ " stands for element-wise "greater than 0 ".

\footnotetext{
${ }^{2}$ In this work, as in [11], upper indexes of matrix expressions such as $k$ in $A_{\mathbf{h}}^{k}$ are not powers, but only for indexation purposes.
} 


\subsection{Lyapunov-based domain of attraction estimation for PWATS}

Classical estimates of the domain of attraction of the origin resort to wellknown invariant set ideas such as Lyapunov level sets [19]. The Lyapunov levelset concept can be generalised including prior estimates of the DA. In particular, the following result will be later exploited:

Theorem 1 ([22]). Consider two sets $A, B$, such that $B \subset A$. If $A$ is invariant and there exist $\gamma>0$ and $V(x)$, bounded in $A$, such that $\dot{V}(x)<-\gamma$ for all $x \in(A-B)$, where $A-B:=\{x \mid x \in A, x \notin B\}$, then all trajectories starting in $A$ enter $B$ in finite time.

LMIs in stability analysis of TS systems usually resort to expressions of the form $A_{i}^{T} P+P A_{i}<0$. Let us review some already-known stability results for PWATS systems.

Defining an augmented state and augmented matrices:

$$
\bar{x}:=\left[\begin{array}{l}
x \\
1
\end{array}\right], \bar{A}_{i}^{k}:=\left[\begin{array}{cc}
A_{i}^{k} & b_{i}^{k} \\
0 & 0
\end{array}\right], i \in\{1,2, \ldots, r\}, k \in K_{1} .
$$

the PWATS stability analysis in [11] can be straightforwardly applied if $\Omega_{k}$ conform to a polyhedral partition of the operating region in the state space.

To see this, consider PWQLFs of the form

$$
V(x):=\bar{x}^{T} \bar{P}_{k} \bar{x}, \quad x \in \Omega_{k},
$$

so that $V(0)=0$, with continuity of the Lyapunov function across the boundaries, i.e., $V_{k}(x(t))=V_{l}(x(t)), \forall x(t) \in\left(\Omega_{k} \cap \Omega_{l}\right)$, guaranteed by parameterising $\bar{P}_{k}$ as

$$
\bar{P}_{k}:=\bar{F}_{k}^{T} T \bar{F}_{k},
$$

where $T$ is a symmetric matrix of adequate dimensions, $\bar{F}_{k}=\left[\begin{array}{ll}F_{k} & f_{k}\end{array}\right]$ with $f_{k}=0$ for $k \in K_{0}$, satisfying $\bar{F}_{k} \bar{x}=\bar{F}_{l} \bar{x}$ for $x \in\left(\Omega_{k} \cap \Omega_{l}\right), k, l \in\{1,2, \ldots, q\}$. Partition information can be systematically incorporated into the analysis via the S-procedure [3]. Notation $\mathbf{I}_{\gamma}:=\operatorname{blkdiag}(\gamma I, 0)$, and $\mathbf{0}_{\gamma}:=\operatorname{diag}(0,0, \ldots, 0, \gamma)$ will be later used. "blkdiag(.)" stands for a square block-diagonal matrix in 
which the diagonal elements are the matrices in the argument. Thus, the following slight generalisations of $[11,20]$ are given:

Theorem 2. If there exist symmetric matrices $T, U_{k} \succeq 0$, and $W_{k i} \succeq 0$ such that, for a given small $\gamma>0$, the LMIs

$$
\begin{gathered}
\bar{P}_{k}-\bar{E}_{k}^{T} U_{k} \bar{E}_{k} \geq \mathbf{I}_{\gamma} \\
\left(\bar{A}_{i}^{k}\right)^{T} \bar{P}_{k}+\bar{P}_{k} \bar{A}_{i}^{k}+\bar{E}_{k}^{T} W_{k i} \bar{E}_{k} \leq-\Phi_{\gamma}^{k}
\end{gathered}
$$

hold for $i \in\{1,2, \ldots, r\}$, being $\Phi_{\gamma}^{k}=\mathbf{I}_{\gamma}$ if $k \in K_{0}$, and $\Phi_{\gamma}^{k}=\mathbf{0}_{\gamma}$ if $k \in$ $K_{1}$, then $x(t)$ tends to zero exponentially for every continuous differentiable piecewise trajectory in $\Omega=\bigcup_{k=1}^{q} \Omega_{k}$ satisfying the model equations (11) with initial conditions $x_{0} \in V_{\beta}$, where $V_{\beta}:=\{x: V(x)<\beta\}$ is any level set of the piecewise $V(x)$ defined in (13) such that $V_{\beta} \subset \Omega$.

Proof outline. Proof follows standard argumentations: first condition proves $V(x)>\gamma x^{T} x$ in region $\Omega_{k}$, and second one proves $\dot{V}(x) \leq-\gamma x^{T} x$ in regions $\Omega_{k}, k \in K_{0}$, and $\dot{V}(x) \leq-\gamma$ in regions $\Omega_{k}, k \in K_{1}$.

Remark 2. From (13), in regions containing the origin $\left(k \in K_{0}\right), V(x)$ is a standard quadratic form without constant or linear terms. As quadratic forms are positive in cones, only the set of conditions with $\bar{E}_{k}=\left[\begin{array}{ll}E_{k} & 0\end{array}\right]$ are relevant if $k \in K_{0}$. In the original reference [11], conditions (15) were separated in two groups according to $k \in K_{0}$ or $k \in K_{1}$; however, such separation is implicitly considered in $\Phi_{\gamma}$ above. In fact, in a region where $e_{k}=0$ and the model is given by TS representation $\bar{A}_{i}^{k}=\operatorname{blkdiag}\left(A_{i}^{k}, 0\right)$, LMIs (15) would entail the Lyapunov function to be forcedly homogeneous quadratic if $V(0)=0$ were enforced. Due to this reason, such separation between $K_{0}$ and $K_{1}$ will be no longer pursued in this work.

Theorem 2 has been extended to the case of non-polyhedral partitions with circular boundaries in the conference paper [23]. For brevity, it will not be discussed here as it will be a particular case of the proposal in this work. 


\subsection{Farkas Lemma and Positivstellensatz}

The above-reviewed prior results can be understood as proving positiveness of quadratic functions in regions with affine/quadratic boundaries; they are instances of the Positivstellensatz argumentation [25, Theorem 1], which in the quadratic-only case amount to the S-procedure [3], and in the affine-only case are a version of Farkas lemma [26]. Computationally, conditions are posed as linear programming (affine case), LMIs (quadratic case) or generic sum-ofsquares constraints [25]. However, the latter exacerbates the computational cost, so it is intentionally left out of the scope of this paper.

Decision variables $U_{k}$ and $W_{k i}$ are generically known as multipliers. In general, the above multiplier-based conditions are only sufficient for emptiness of semialgebraic sets or for sign-definiteness of some polynomial functions of the state in particular regions ${ }^{3}$.

However, there are a few well-known situations in which exact results can be asserted with few computational resources. These situations are: the Sprocedure with a single quadratic constraint, and the Farkas Lemma for affine constraints (in linear programming setups). The latter can be stated as:

Lemma 1 (Farkas Lemma [26]). Consider an affine function $V(x)=p^{T} x+\delta$, where $p \in \mathbb{R}^{n \times 1}$ and $\delta \in \mathbb{R}$, and a polyhedral region $\Omega:=\{\sigma(x) \succeq 0\}$ being $\sigma(x):=\left[\begin{array}{ll}E & e\end{array}\right] \bar{x}$, where $E \in \mathbb{R}^{N \times n}$ and $e \in \mathbb{R}^{N \times 1}$. Let $\sigma_{l}(x)$ be the $l$-th element of vector $\sigma(x)$. Then, the following expressions are equivalent:

a) $V(x)=p^{T} x+\delta \geq 0$ for all $x \in \Omega$

b) There exist $\tau_{l} \geq 0, l \in\{1,2, \ldots, N\}$ such that

$$
V(x)-\sum_{l=1}^{N} \tau_{l} \sigma_{l}(x) \geq 0, \quad \forall x \in \mathbb{R}^{n}
$$

Corollary 1. Under the same settings, the following expressions are equivalent:

\footnotetext{
${ }^{3}$ More general conditions may be obtained by transforming the multipliers into polynomials of arbitrary degree; however, as pointed out at the introduction, it is at the expense of a heavy computational cost [22].
} 
a) $V(x)=p^{T} x+\delta=0$ for all $x \in \Omega$, and $\Omega \neq \emptyset$.

b) There exist arbitrary $\tau_{l}, l \in\{1,2, \ldots, N\}$ such that

$$
V(x)-\sum_{l=1}^{N} \tau_{l} \sigma_{l}(x)=0, \quad \forall x \in \mathbb{R}^{n}
$$

Proof. See Appendix.

In the next sections, earlier results will be generalised using the ideas in Sections 2.3 and 2.2; asymptotical exactness of the proposed approach will be established via universal-approximation argumentations.

\section{Main Results}

Let us consider a connected modelling region $\Omega$ partitioned into $q$ subregions $\Omega_{k}$ with disjoint interiors where each region is defined ${ }^{4}$ as:

$$
\Omega_{k}=\left\{x: E_{k} \bar{x} \succeq 0, \bar{x}^{T} Q_{l k} \bar{x} \geq 0, l \in\left\{1,2, \ldots, \ell_{k}\right\}\right\}
$$

where $\bar{x}$ is obtained from $x$ using (12). The $j$-th affine constraint, corresponding to the $j$-th row of $E_{k}$ will be denoted as $E_{j k}{ }^{5}$. The "faces" of $\Omega_{k}$ will be defined by changing just one of the affine or quadratic inequalities to equality.

If $Q_{l k}=0$, or, equivalently, $\ell_{k}=0$, the partition will be said to be polyhedral. Given that the regions have disjoint interior by assumption, the intersection of two regions $\Omega_{k}$ and $\Omega_{l}$ must be a subset of a face in each of them. The region $\Omega_{k}$ will have a number of vertices located at the intersection of $n$ faces.

\footnotetext{
${ }^{4}$ For notational simplicity, denoting constraints associated to regions containing the origin with $E_{k}$, and those where $0 \notin \Omega_{k}$ with $\bar{E}_{k}$ (established in [11]), will no longer be used. All matrices in (18) will be assumed to apply on the extended state $\bar{x}$. In this way cluttering all matrices with barred notation is avoided while leaving $\bar{E}$ available for future definitions.

${ }^{5}$ Following notation in [11], indexes will be stacked together in order to avoid long expressions; system matrices will use upper and lower ones.
} 


\subsection{Continuity in the Piecewise Lyapunov Function}

Continuity of the piecewise Lyapunov function was enforced via (14) in prior works. A more flexible alternative will be proposed next. Consider a non-empty set $\mathcal{X}:=\left\{\bar{x}: E \bar{x}=0, \bar{x}^{T} Q_{1} \bar{x}=0, \bar{x}^{T} Q_{2} \bar{x}=0, \ldots, \bar{x}^{T} Q_{\bar{\ell}} \bar{x}=0\right\}$, such that $\Omega_{k} \cap$ $\Omega_{m} \subset \mathcal{X}$, for some $k, m$.

Lemma 2. The piecewise quadratic function

$$
V(x)=\left\{\begin{array}{l}
\bar{x}^{T} \bar{P}_{k} \bar{x} \text { for } x \in \Omega_{k}, \\
\bar{x}^{T} \bar{P}_{m} \bar{x} \text { for } x \in \Omega_{m},
\end{array}\right.
$$

is continuous in the "face" $\Omega_{k} \cap \Omega_{m}$ if, given $\mathcal{X}$ in the above form such that $\Omega_{k} \cap \Omega_{m} \subset \mathcal{X}$, there exists an arbitrary multiplier matrix $U$ and arbitrary scalars $\tau_{j}$ such that:

$$
\bar{P}_{k}-\bar{P}_{m}+U E+E^{T} U+\sum_{j=1}^{\bar{\ell}} \tau_{j} Q_{j}=0
$$

Proof. Since $0=\bar{x}^{T}\left(\bar{P}_{k}-\bar{P}_{m}+U E+E^{T} U+\sum_{j=1}^{\bar{\ell}} \tau_{j} Q_{j}\right) \bar{x}=\bar{x}^{T}\left(\bar{P}_{k}-\bar{P}_{m}\right) \bar{x}$ for $\bar{x} \in \mathcal{X}$, then the result is trivial.

In this way, matrices $F$ and decision variables $T$ parameterising the sought Lyapunov functions, used in prior literature, are not needed in this proposal, giving more clarity and flexibility, in exchange for additional multipliers.

Remark 3. Note that, from analytical prolongation (or Taylor series), if two functions coincide on an infinitesimal fragment of a face (i.e., a small lowerdimensional affine or quadratic region), they do on all prolongations. This is the reason of considering the above set $\mathcal{X}$ which disregards inequalities in $\Omega_{k} \cap \Omega_{m}$ (for instance, with $\Omega_{1}=\left\{9-x^{T} x \geq 0, x^{T} x-1 \geq 0, x_{2} \geq 0\right\}, \Omega_{2}=$ $\left\{1-x^{T} x \geq 0, x_{2} \geq 0\right\}$, we would have that $\Omega_{1} \cap \Omega_{2}=\left\{1-x^{T} x=0, x_{2} \geq 0\right\}$, and $\mathcal{X}=\left\{1-x^{T} x=0\right\}$; adding a multiplier associated to constraint $x_{2} \geq 0$ would be useless). 


\subsection{Extension of piecewise quadratic stability analysis}

In Theorem 2, taken from [11], only multipliers $U_{k}$ in $E_{k}^{T} U_{k} E_{k}$ (and $W_{k i}$, with the same role) appeared to enforce local positiveness (negativeness) of the Lyapunov function (and its derivative).

However, we can state a more general condition.

Lemma 3. Consider the set

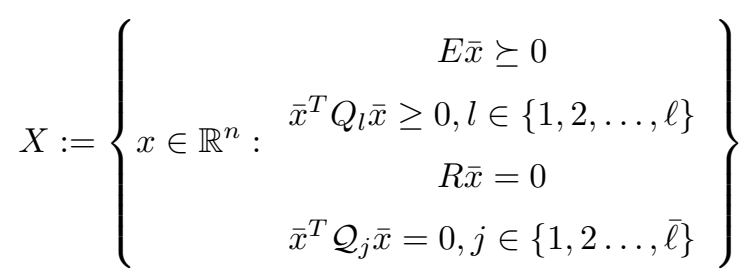

Consider, too, a quadratic polynomial $\bar{x}^{T} \Xi \bar{x}$. Then, $\bar{x}^{T} \Xi \bar{x} \geq 0$ for all $x \in X$ if there exist arbitrary scalars $\xi_{j}, j \in\{1,2, \ldots, \bar{\ell}\}$, arbitrary matrix $Z$, positive scalars $\tau_{l}, l \in\{1,2, \ldots, \ell\}$, and element-wise positive matrix $U$ such that the following matrix inequality holds:

$$
-\Xi+\sum_{l=1}^{\ell} \tau_{l} Q_{l}+\bar{E}^{T} U \bar{E}+\sum_{j=1}^{\bar{\ell}} \xi_{j} \mathcal{Q}_{j}+Z^{T} R+R^{T} Z \leq 0
$$

where ${ }^{6}$

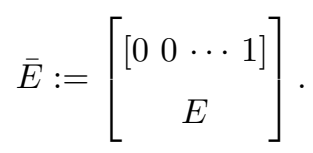

Proof. Indeed, for any $x \in X$, we have $\sum_{l=1}^{\ell} \tau_{l} Q_{l}+\bar{E}^{T} U \bar{E}+\sum_{j=1}^{\bar{\ell}} \xi_{j} \mathcal{Q}_{j}+$ $Z^{T} R+R^{T} Z \geq 0$. Hence, if (20) holds, it proves that $-\bar{x}^{T} \Xi \bar{x} \leq 0$ in $X$, i.e., $\bar{x}^{T} \Xi \bar{x} \geq 0$.

Corollary 2. Letting $\Xi=\operatorname{diag}(0,0, \ldots, 0,-1)$, if there exists the above-mentioned multipliers then $X$ is empty.

Proof. Indeed, we proved $0 \geq 1$ on $X$ so forcefully $X$ should be empty.

\footnotetext{
${ }^{6}$ Recall $\bar{E}$ carrying the meaning in [11] is henceforth no longer in use.
} 
Corollary 3. If $\bar{x}^{T} \Xi \bar{x}$ is a degree-1 polynomial, and $X$ is a full-dimensional polyhedron $\left(Q_{l}=\mathcal{Q}_{j}=0, R=0\right)$, then conditions in Lemma 3 are necessary and sufficient.

Proof. It can be shown that the choice of multipliers encompasses those in Farkas lemma, i.e., the multipliers $\tau_{l}$ in (16) from Lemma 1. Details omitted for brevity.

Remark 4. The fact that the last element of $\bar{x}$ is equal to 1 , as well as the seemingly "trivial" addition of $1 \geq 0$ in the construction of $\bar{E}$, introduces additional multipliers, which were not considered in prior literature; this enables the above generalisation and exactness in the affine case (Corollary 3). Without $\bar{E},(20)$ cannot be written as (16) in the polyhedral case $\left(Q_{l}=Q_{j}=0\right)$. Apart, combined affine/quadratic boundaries are considered, as well as equalities which do not appear in (18), but will be relevant when geometric conditions are pursued.

Consider now a PWATS model (11) defined over a quadratic/polyhedral partition of a region $\Omega$ with sets $\Omega_{k}=\left\{x: \sigma_{j}^{k}(x) \geq 0, j \in\left\{1,2, \ldots, n_{k}\right\}\right\}$, $k \in\{1,2, \ldots, q\}$ defined as (18), i.e. being each of the constraints $\sigma_{j}^{k}(\cdot)$ either affine or quadratic.

The following definition will single out constraints which take part in the shape of the overall modelling region $\Omega=\cup_{k} \Omega_{k}$ defining its outer boundary:

Definition 1 . The face generated by constraint $\sigma_{j}^{k}(\cdot)$ will be denoted as:

$$
\mathcal{F}_{j}^{k}:=\left\{x: \sigma_{j}^{k}(x)=0\right\} \cap \Omega_{k}
$$

Such face (and the constraint $\sigma_{j}^{k}$ itself) is called "outer" if:

$$
\mathcal{F}_{j}^{k} \not \subset \bigcup_{l \neq k} \Omega_{l}
$$

An illustration of the meaning of the above definition appears on Figure 1, where outer faces are labelled with $\mathcal{F}_{\text {out }}^{k}, k=\{1,2,3,4\}$.

Obviously, the boundary $\partial \Omega$, fulfills $\partial \Omega \subset \bigcup_{\mathcal{F}_{j}^{k} \text { is outer }} \mathcal{F}_{j}^{k}$. 


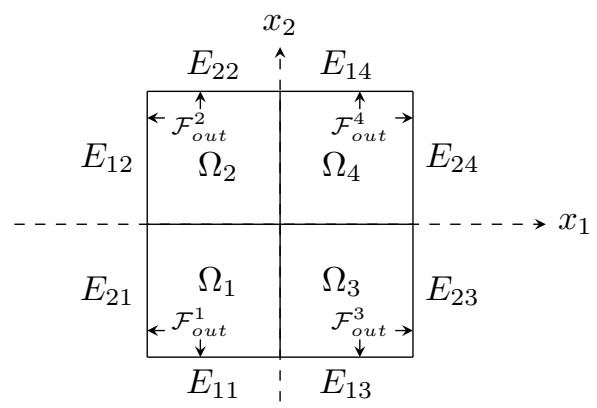

Figure 1: Bounding hyperplanes $\mathcal{F}_{\text {out }}^{k}$ delimiting $\Omega$.

Let us denote as $\partial \Omega^{\downarrow}$ as the set of points in the boundary of $\Omega$ such that system trajectories which contain them "enter" $\Omega$, i.e., in formal terms:

$$
\partial \Omega^{\downarrow}:=\{x \in \partial \Omega: \exists h>0 \text { s.t. } \phi(\epsilon, x) \in \Omega \forall 0<\epsilon<h\}
$$

Let us denote as $\partial \Omega^{\uparrow}$ the complementary of $\partial \Omega^{\downarrow}$ in $\partial \Omega$, i.e., the points in the boundary of $\Omega$ such that trajectories do not immediately enter the interior of $\Omega$.

For later use, we will denote the set of all outer constraints as:

$$
\mathcal{I}_{k}:=\left\{j: \sigma_{j}^{k}(\cdot) \text { is outer }\right\}
$$

Given an arbitrary point $x \in \partial \Omega_{k}$, let us denote as $\Gamma_{k}(x)$ the set of outer constraints in $\Omega_{k}$ which are active at $x$, i.e., the ones associated to the outer faces $x$ belongs to:

$$
\Gamma_{k}(x):=\left\{j \in \mathcal{I}_{k}: \sigma_{j}^{k}(x)=0\right\}
$$

Proposition 1. Given $x \in \partial \Omega_{k} \cap \partial \Omega$, if $\dot{\sigma}_{j}^{k}(x)>0$ for all $j \in \Gamma_{k}(x)$, then $x \in \partial \Omega^{\downarrow}$. Proof. First, note that, for the active constraints $\sigma_{k}^{k}(x)=0, \dot{\sigma}_{j}^{k}(x)>0$ entails $\sigma(\phi(\epsilon, x))>0$ for all $\epsilon$ such that $0<\epsilon<h$ for small enough $h$. Given that $\sigma(x)>0$ for inactive constraints, then for small enough $h, \sigma(\phi(\epsilon, x))>0$ will still hold for such constraints for all $0<\epsilon<h$. Hence, no other constraint will be active and all active ones will render inactive: $\phi(\epsilon, x)$ will belong to the interior of $\Omega$. 
Consider, given $x$ and the constraints indexed in $\Gamma_{k}(x)$, that a particular active constraint is either affine $\sigma_{j}^{k}(x)=E_{j k} \bar{x}$, being $E_{j k}$ a row vector, or quadratic $\sigma_{j}^{k}(x)=\bar{x}^{T} Q_{j k} \bar{x}$, being $Q_{j k}$ a matrix of adequate size.

Corollary 4. Given $x \in \partial \Omega_{k} \cap \partial \Omega$, if, for all $i \in\{1,2, \ldots r\}$, for all $j \in \Gamma_{k}(x)$ either:

- $E_{j k} \bar{A}_{i}^{k} \bar{x}>0$, if $\sigma_{j}^{k}(\cdot)$ is affine, or

- $\bar{x}^{T}\left(Q_{j k} \bar{A}_{i}^{k}+\left(\bar{A}_{i}^{k}\right)^{T} Q_{j k}^{T}\right) \bar{x}>0$ if $\sigma_{j}^{k}(\cdot)$ is quadratic,

then $x \in \partial \Omega^{\downarrow}$.

Proof. The conditions on the vertices of the PWATS model are sufficient to ensure that conditions in Proposition 1 hold, as $\dot{\bar{x}}$ belongs to the convex hull of the vertex derivative estimates $\bar{A}_{i}^{k} \bar{x}$.

Now, we are in conditions to state the main result of the paper:

Theorem 3. Consider a nonlinear system (1), and a PWATS model (11) of $i t$, defined over a partition of a compact region $\Omega$ with sets $\Omega_{k}, k \in\{1,2, \ldots, q\}$ defined as in (18). Consider, too, a collection of ellipsoids $\mathcal{E}_{s}^{k}=\left\{x: \bar{x}^{T} \bar{G}_{k s} \bar{x}>\right.$ $0\}$ for $s \in\left\{1,2, \ldots, \bar{s}_{k}\right\}$, such that $\mathcal{E}_{s}^{k} \cap \Omega_{k}$ belongs to the $D A$ of $x=0$ for the nonlinear system (1), and a second collection of ellipsoids $\hat{\mathcal{E}}_{j s}^{k}=\left\{x: \bar{x}^{T} \hat{G}_{k j s} \bar{x}>\right.$ $0\}, s \in\left\{1,2, \ldots, \hat{s}_{k j}\right\}$, associated to each face $\mathcal{F}_{j}^{k}$ such that $\hat{\mathcal{E}}_{s}^{k} \cap \mathcal{F}_{j}^{k}$, too, belongs to the DA of $x=0$. Then, if there exist symmetric matrices $\bar{P}_{k}$ satisfying the continuity conditions ${ }^{7}$

$$
\bar{x}^{T} \bar{P}_{k} \bar{x}=\bar{x}^{T} \bar{P}_{m} \bar{x}, \forall x \in\left(\Omega_{k} \cap \Omega_{m}\right),
$$

symmetric matrices $U_{k i}^{1} \succeq 0, U_{k j i}^{2} \succeq 0$, arbitrary row vectors $Z_{j k}$, positive scalars $\tau_{k l}^{1}, \tau_{k s}^{2}, \tau_{k l}^{3}, \tau_{k s}^{4}, \tau_{k j i}^{5}, \tau_{k j s}^{6}, i \in\{1,2, \ldots, r\}$, and arbitrary scalars $\tau_{k j}^{7}, j \in \mathcal{I}_{k}$, $m \in\{1,2, \ldots, q\}$, yielding a feasible solution for the following inequalities, given

\footnotetext{
${ }^{7}$ Which can be enforced via LMI conditions (19) on all shared faces.
} 


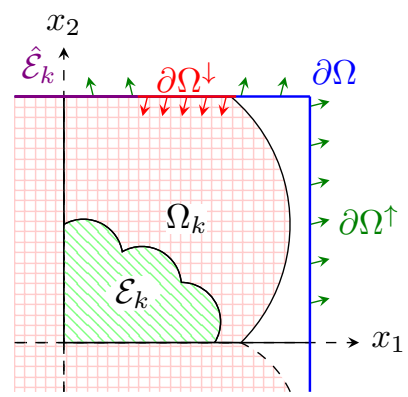

Figure 2: Subsets $\Omega_{k}, \mathcal{E}_{k}, \hat{\mathcal{E}}_{k}, \partial \Omega$, and $\partial \Omega^{\downarrow}$.

$\gamma>0$, first:

$$
\bar{P}_{k} \bar{A}_{i}^{k}+\left(\bar{A}_{i}^{k}\right)^{T} \bar{P}_{k}+\bar{E}_{k}^{T} U_{k i}^{1} \bar{E}_{k}+\sum_{l=1}^{\ell_{k}} \tau_{k l}^{1} Q_{l k}-\sum_{s=1}^{\bar{s}_{k}} \tau_{k s}^{2} \bar{G}_{s k} \leq-\Phi_{\gamma}^{k}
$$

being $\Phi_{\gamma}^{k}=\mathbf{I}_{\gamma}$ if $k \in K_{0}$, and $\Phi_{\gamma}^{k}=\mathbf{0}_{\gamma}$ if $k \in K_{1}$;

and, second, either, if $\sigma_{j}^{k}=E_{j k} \bar{x}$ (affine constraints):

$$
Z_{j k}^{T} E_{j k}+(*)+\bar{P}_{k}-\mathbf{E}_{k j i}^{T} U_{k j i}^{2} \mathbf{E}_{k j i}-\sum_{l=1}^{\ell_{k}} \tau_{k l}^{3} Q_{l k}+\sum_{s=1}^{\bar{s}_{k}} \tau_{k s}^{4} \bar{G}_{s k}+\sum_{s=1}^{\hat{s}_{k j}} \tau_{k j s}^{6} \hat{G}_{k j s} \geq 0,
$$

where

$$
\mathbf{E}_{k i j}=\left[\begin{array}{c}
\bar{E}_{k} \\
-E_{j k} \bar{A}_{i}^{k}
\end{array}\right] ;
$$

or, if $\sigma_{j}^{k}=\bar{x}^{T} Q_{j k} \bar{x}$ (quadratic constraints):

$$
\begin{aligned}
\tau_{k j}^{7} Q_{j k}+\bar{P}_{k}-\bar{E}_{k}^{T} U_{k j i}^{2} \bar{E}_{k}+ & \tau_{k j i}^{5}\left(Q_{j k} \bar{A}_{i}^{k}+\left(\bar{A}_{i}^{k}\right)^{T} Q_{j k}^{T}\right) \\
& -\sum_{l=1}^{\ell_{k}} \tau_{k l}^{3} Q_{l k}+\sum_{s=1}^{\bar{s}_{k}} \tau_{k s}^{4} \bar{G}_{s k}+\sum_{s=1}^{\hat{s}_{k j}} \tau_{k j s}^{6} \hat{G}_{k j s} \geq 0
\end{aligned}
$$

then, $\left\{x: \bar{x}^{T} \bar{P}_{k} \bar{x}<0\right\} \cap \Omega_{k}$ belongs to the $D A$ of $x=0$ for every $k$, for the nonlinear system under study.

Proof. See Appendix.

Remark 5. Theorem 3 requires a prior estimate of the DA of the origin $\mathcal{E}$. In order to apply the above result to prove stability of a PWATS model without 


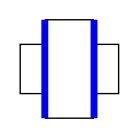

$(\mathrm{P} 1)$

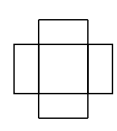

(P2)

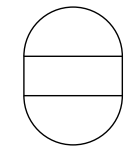

(P3)

Figure 3: Example partitions: (P1) is not a honeycomb; (P2,P3) are.

such "initialisation" (to get results with the same a priori assumptions as usual literature), the theorem should be modified by setting $\bar{G}_{k s}=0$, thus initialising the ellipsoids $\mathcal{E}_{s}^{k}$ to empty sets (equivalently, forgetting about the terms with $G$ in the LMIs, letting $\left.\bar{s}_{k}=0\right)$. The result is as follows.

Corollary 5. A PWATS model (11), defined over a partition of a region $\Omega$ with sets $\Omega_{k}, k \in\{1,2, \ldots, q\}$ defined as in (18), is locally stable if there exist decision variables fulfilling Theorem 3 with $\bar{s}_{k}=0$ and $\hat{s}_{k j}$, such that the set $\mathcal{V}$ in (A.3) is not empty.

Proof. Indeed, applying the prior theorem, $\left\{x: \bar{x}^{T} \bar{P} \bar{x}<0\right\} \cap \Omega$ belongs to the DA of $x=0$ and, by assumptions in the corollary statement, it is not empty. In this particular situation, contrarily to footnote 12 , the set $\mathcal{W}_{\boldsymbol{\varepsilon}}$ would be empty, and $\mathcal{W}=\mathcal{V}=\mathcal{W}_{\neg \varepsilon}$, actually containing the origin, deduced with an identical argumentation to the one in the theorem's proof for this particular case $\mathcal{E}=\emptyset$.

Note that non-emptiness of $\mathcal{V}$ can be enforced in the LMI conditions with some geometric conditions. This is the objective of next subsection.

In order to avoid conservatism, we will assume that the chosen partition conforms a honeycomb [27], defined as a partition where vertices of the regions are common to neighboring ones (a region $\Omega_{j}$ will be understood to be neighboring to $\Omega_{k}$ if $\Omega_{j} \cap \Omega_{k} \neq \emptyset$, int $\left(\Omega_{j}\right) \cap \operatorname{int}\left(\Omega_{k}\right)=\emptyset$; vertices will be the points formed by intersection of $n$ faces).

For instance, Figure 3 shows a partition (P1) which does not fulfill the honeycomb assumption, and a pair of another ones which do. The reason of such assumption is that the faces of the central region in partition (P1) (marked as 
a thick blue line) are outer, so the theorem would preclude a level set including the subset of the face where trajectories enter the neighboring regions, which is clearly undesired. The second partition (P2) is a honeycomb and such issue does not appear. Partition (P3) is, too, a honeycomb with quadratic boundaries.

\subsection{Geometric optimisation}

In order for the theorem to be useful, some additions enforcing how to obtain the "largest" estimate of the domain of attraction should be added, for instance, maximising the size of some prefixed-shape set which can be fit inside the obtained DA estimate (via maximisation of scaling factors).

Consider a prefixed-shape region in the form:

$$
\hat{\Omega}:=\left\{x: \tilde{E} \bar{x} \succeq 0, \bar{x}^{T} \tilde{Q}_{1} \bar{x} \geq 0, \ldots, \bar{x}^{T} \tilde{Q}_{\tilde{q}} \bar{x} \geq 0\right\}
$$

where some affine inequalities (rows of $\tilde{E}$ ) and $\tilde{q}$ quadratic ones hold. Let us define the geometric transformation below:

$$
\bar{x}_{\lambda}:=\left[\begin{array}{c}
x_{c}+\lambda^{-1}\left(x-x_{c}\right) \\
1
\end{array}\right]=\Lambda \bar{x}
$$

where $\Lambda:=\left[\begin{array}{cc}\lambda^{-1} I & x_{c}-\lambda^{-1} x_{c} \\ 0 & 1\end{array}\right]$, being $\lambda$ a "scaling factor" and $x_{c}$ a "scaling centroid", both parameters assumed known. The scaled region $\tilde{\Omega}$ by factor $\lambda$ around $x_{c}$ is defined as:

$$
\tilde{\Omega}(\lambda):=\left\{x: \tilde{E} \Lambda \bar{x} \succeq 0, \bar{x}^{T} \Lambda^{T} \tilde{Q}_{1} \Lambda \bar{x} \geq 0, \ldots, \bar{x}^{T} \Lambda^{T} \tilde{Q}_{\tilde{q}} \Lambda \bar{x} \geq 0\right\}
$$

Note that setting $x_{c}=0$ reduces the scaling to the standard scaling around the origin.

Theorem 4. Consider a PWATS model (11) defined over a partition of a region $\Omega$ with sets $\Omega_{k}, k \in\{1,2, \ldots, q\}$ defined as in (18). Consider, too, a collection of ellipsoids $\mathcal{E}_{s}^{k}=\left\{x: \bar{x}^{T} \bar{G}_{s k} \bar{x}>0\right\}$ for $s \in\left\{1,2, \ldots, \bar{s}_{k}\right\}$, such that $\mathcal{E}_{s}^{k} \cap \Omega_{k}$ belongs to the DA of $x=0$ for the nonlinear system (1), and a second collection of ellipsoids $\hat{\mathcal{E}}_{j s}^{k}=\left\{x: \bar{x}^{T} \hat{G}_{k j s} \bar{x}>0\right\}, s \in\left\{1,2, \ldots, \hat{s}_{k j}\right\}$, associated to each 
face $\mathcal{F}_{j}^{k}$ such that $\hat{\mathcal{E}}_{s}^{k} \cap \mathcal{F}_{j}^{k}$, too, belongs to the DA of $x=0$. Then, if there exist symmetric matrices $\bar{P}_{k}, U_{k i}^{1} \succeq 0, U_{k j i}^{2} \succeq 0, U_{k}^{3} \succeq 0, U_{k}^{4} \succeq 0$, arbitrary column vectors $Z_{j k}$, arbitrary scalars $\tau_{k j}^{7}$, and positive scalars $\tau_{k l}^{1}, \tau_{k s}^{2}, \tau_{k l}^{3}, \tau_{k s}^{4}$, $\tau_{k j i}^{5}, \tau_{k j s}^{6}, \tau_{k^{\prime} l}^{8}, \tau_{k^{\prime} m}^{9}, \tau_{k^{\prime} s}^{10}, i \in\{1,2, \ldots, r\}, j \in \mathcal{I}_{k}, m \in\{1,2, \ldots, \hat{q}\}$, yielding a feasible solution for the inequalities (24), either (25) or (26), (28), and, for a given $k^{\prime}$, and $\gamma>0$ :

$$
\begin{aligned}
\bar{P}_{k^{\prime}}+\bar{E}_{k^{\prime}}^{T} U_{k^{\prime}}^{3} \bar{E}_{k^{\prime}}+\Lambda^{T} \tilde{E}_{k^{\prime}}^{T} U_{k^{\prime}}^{4} \tilde{E}_{k^{\prime}} \Lambda+\sum_{l=1}^{\ell_{k^{\prime}}} \tau_{k^{\prime} l}^{8} Q_{l k^{\prime}} \\
+\sum_{m=1}^{\hat{q}} \tau_{k^{\prime} m}^{9} \Lambda^{T} \tilde{Q}_{m} \Lambda-\sum_{s=1}^{\bar{s}_{k^{\prime}}} \tau_{k^{\prime} s}^{10} \bar{G}_{s k^{\prime}} \leq-\mathbf{0}_{\gamma}
\end{aligned}
$$

then, the region $\hat{\Omega}(\lambda) \cap \Omega_{k^{\prime}}$ belongs to the $D A$ of $x=0$.

Proof. See Appendix.

Note that $\hat{\mathcal{E}}$ has not been used in conditions (30); indeed, such $\hat{\mathcal{E}}$ is formed by fragments of outer faces with no volume, but $\hat{\Omega}(\lambda) \cap \Omega_{k^{\prime}}$ will have nonzero volume except in degenerate cases, so behaviour at the faces is irrelevant for the level sets of $\bar{P}_{k^{\prime}}$ in $\Omega_{k}$.

Remark 6. The above theorem can be extended to forcing shape constraints in several regions, by repeating (30) for different $k^{\prime}$ in a selected set (or even all of them). The fixed-shape conditions above can be particularised to spherical regions, polytopes (boxes), or intersections thereof, extending analogous geometrical conditions in LMI setups for classical (non-affine) TS systems [3, 28].

Remark 7. Theorem 4 provides only feasibility conditions. Trivially, they can be converted to optimisation ones on the centroid/size "shape" parameters $\left(x_{c}\right.$, ג). If only one of them is to be optimised (either scale or translation), such optimisation setups can be cast as bisection problems and, in some particular cases as GEVP ones or even LMI ones in Lyapunov and shape parameters. Such developments are transcriptions to the affine case of well-studied geometric 


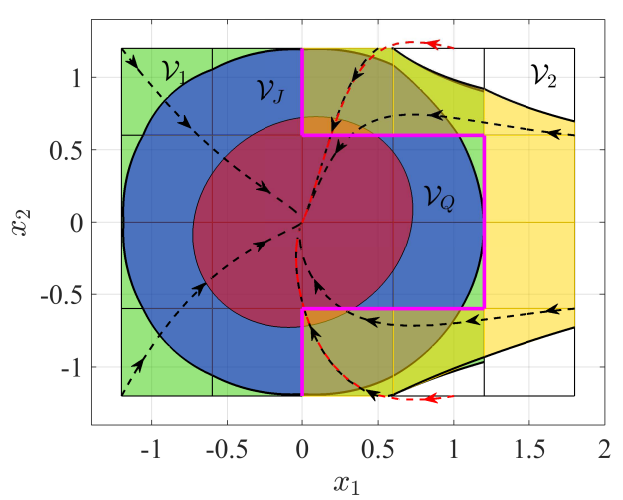

Figure 4: Estimation of the DA for example 1: quadratic TS case (region in red, [28]), Thm. 2 (region in blue, [20]), and Thm. 4 (region in green). Yellow region also depicts the result of a second execution of Theorem 4 only on the squares at the right of the magenta boundary, seeding it with the prior green region.

problems $^{8}$ and are omitted for brevity, leaving details to particular examples later.

The following corollary shows that our result extends prior literature.

Corollary 6. In the polyhedral partition case, if LMIs in Theorem 2 are feasible, and $\Omega$ contains a neighborhood of the origin, then conditions on Corollary 5 hold for some non-empty domain of attraction.

Proof. See Appendix.

Our proposal, apart from giving the same (or better) solutions as Theorem 2 in an identical setting, improving over [11, 20], applies to regions with quadratic boundaries, it is less conservative (due to $\bar{E}$, and to the fact that the level set can get "out" of $\Omega$ ) and, last, $\Omega$ can even not contain the origin as long as a fraction of it is proven (elsewhere) to belong to the DA of the origin.

\footnotetext{
${ }^{8}$ For instance, the smallest or largest circle inside an ellipsoid, the largest ellipsoid inside a polytope, etc. in [3].
} 
Example $1([22])$. Consider the following nonlinear system:

$$
\dot{x}_{1}=0.5 x_{2}-3 x_{1}, \quad \dot{x}_{2}=\left(3 \sin x_{1}-2\right) x_{2}
$$

where the state is assumed to lie in the compact set $\Omega=\left\{x:\left|x_{i}\right| \leq 1.2, i=1,2\right\}$. Consider a partition of the compact set $\Omega$ in $q=16$ subsets, as it is shown in Figure 4. An initial estimation of the DA was obtained using a quadratic Lyapunov function and a standard 2-rule TS model resulting from choosing $\rho(x)=3 \sin \left(x_{1}\right) x_{2}$, computed in a smaller modelling region $\Omega_{T S}=\left\{x:\left|x_{i}\right| \leq\right.$ $0.72, i=1,2\}$. The resulting largest level set in $\Omega_{T S}$ is given by $\mathcal{V}_{Q}=\{x$ : $\left.x^{T} \bar{P}_{Q} x<0\right\}$, with:

$$
\bar{P}_{Q}=\left[\begin{array}{ccc}
1.9104 & -0.2365 & 0 \\
-0.2365 & 1.9104 & 0 \\
0 & 0 & -1
\end{array}\right] .
$$

Such level set is depicted in red in the referred figure.

Now, a PWATS model has been generated with the same choice of $\rho(x)$ applying the optimisation setup discussed in [10].Theorem 4 was applied in order to find the largest circle $\tilde{\Omega}(\lambda)=\left\{x:-\lambda^{-1} x^{T} x+1 \geq 0\right\}$ inside the proven domain of attraction, minimising $\lambda^{-1}$ by bisection, stating conditions (30) for all the regions. The knowledge that the red region already belonged to the DA has been exploited in the LMI conditions. In Figure 4, the larger resulting level-set $\mathcal{V}$ is shown in green. The level set intersects with the frontier of $\Omega$, as the theorem allows for it; the only regions out of it are the top and bottom right white zones.

For comparison, a estimation of the DA using classical Theorem 2 for the same PWATS model is shown in blue. In this case, level sets from earlier results cannot exit $\Omega$.

Last, the 8 squares containing the yellow regions in the figure are used in a new estimation of the DA with a partition which does not contain the origin but contains as initial DA estimates both the prior green piecewise-ellipsoidal fragments conforming $\mathcal{E}$ and the magenta lines conforming $\hat{\mathcal{E}}$. With the same geometric objective, the referred yellow region can be proved to belong to the 
domain of attraction ${ }^{9}$. Some simulated trajectories show that, indeed, the DA estimate is correct.

\subsection{Iterative Enlargement of the Domain of Attraction}

The basic idea in this section is proving a large DA estimate by modifying $\Omega$ as the region proved with Theorem 4 grows larger, removing "empty" regions (in order to be less conservative at next iteration), and adding new neighboring regions around the ones that contain any points in the proven DA, i.e. around those in which there exists an ellipsoid $\mathcal{E}_{s}^{k}$ such that $\mathcal{E}_{s}^{k} \cap \Omega_{k} \neq \emptyset$. In order to carry out such operation, the following result will be used:

Lemma 4. Consider a region $\Omega_{k}$ defined as in (18) and a collection of ellipsoids $\mathcal{E}_{s}^{k}=\left\{x: \bar{x}^{T} \bar{G}_{k s} \bar{x}>0\right\}$ for $s \in\left\{1,2, \ldots, \bar{s}_{k}\right\}$. Then, the two assertions below are true:

a) if $\exists \tau_{s}^{1} \geq 0, \tau_{l}^{2} \geq 0, U=U^{T} \succeq 0$ such that $\mathbf{0}_{1}-\sum_{l=1}^{\bar{s}_{k}} \tau_{s}^{1} \bar{G}_{k s}+\hat{E}_{k}^{T} U \hat{E}_{k}+$ $\sum_{l=1}^{\ell_{k}} \tau_{l}^{2} Q_{l k} \leq 0$, then $\Omega_{k} \subset \cup_{s}^{\bar{s}_{k}} \mathcal{E}_{s}^{k}$.

b) if $\exists \tau_{s}^{1} \geq 0, \tau_{l, s}^{2} \geq 0, U_{s}=U_{s}^{T} \succeq 0$ such that, for all $s, \mathbf{0}_{1}+\tau_{s}^{1} \bar{G}_{k s}+\hat{E}_{k}^{T} U_{s} \hat{E}_{k}+$ $\sum_{l=1}^{\ell_{k}} \tau_{l, s}^{2} Q_{l k} \leq 0$, then $\Omega_{k} \cap \cup_{s}^{\bar{s}_{k}} \mathcal{E}_{s}^{k}=\emptyset$.

Proof. The first condition a) proves that $\Omega_{k} \cap\left(\cap_{s=1}^{\bar{s}_{k}}\left\{x: \bar{x}^{T} \bar{G}_{k s} \bar{x} \leq 0\right\}\right)$ is empty from Corollary 2, and therefore $\Omega_{k} \subset \cup_{s}^{\bar{s}_{k}} \mathcal{E}_{s}^{k}$, because $\cap_{s=1}^{\bar{s}_{k}}\left\{x: \bar{x}^{T} \bar{G}_{k s} \bar{x} \leq 0\right\}$ is the set of $\bar{x}$ lying outside the union of the ellipsoids $\mathcal{E}_{s}^{k}$.

The second condition b) proves that $\Omega_{k} \cap \mathcal{E}_{s}^{k}=\emptyset$ for every $s$, from Corollary 2, and, hence, so it is $\Omega_{k} \cap \cup_{s}^{\bar{s}_{k}} \mathcal{E}_{s}^{k}=\emptyset$.

If the ellipsoids are those in Theorem 3, Lemma 4 ensures that regions fulfilling the first LMI have been totally proven to belong to the DA, and regions fulfilling the second set of LMIs (one for each $s$ ) have no point in them proven to belong to the DA. The former ones will be labelled as "full" and the latter ones, as "empty".

\footnotetext{
${ }^{9}$ Actually, as complete faces are in the DA, instead of being considered in $\hat{\mathcal{E}}_{k}$, they can be equivalently removed from the set of outer faces, details omitted for brevity.
} 


\subsubsection{Algorithm}

Based on Theorem 3 and the discussed idea above, Algorithm 1 on top of next page is proposed, initialising on a prior feasible solution and iteratively improving the DA estimate by suitably modifying the partition (adding, removing and dividing regions). Some remarks are presented below detailing the ideas in some of its steps.

Remark 8. [Initialization] The algorithm will be initialised with any piecewise partition of an initial compact set $\Omega^{[0]}$ where a PWQLF has been obtained via a feasible solution of any LMI in literature, for instance:

- a single region with a TS model, as done in Example 1,

- a feasible piecewise-quadratic DA estimate from Johansson's Theorem 2 or, better,

- a solution from Corollary 5 (with some geometric optimisation, Theorem 4) with initial empty DA estimate, proved to be more general than Theorem 2.

Remark 9. [Neighbouring region generation] Depending on the geometry of the chosen partition (simplicial, parallelotopic, etc.), generating these new neighbouring regions might require different code implementations; in later examples, a particular hyper-cube-based setting will be explained, based on the fact that a space-filling tessellation is possible with congruent copies of any parallelotope.

Remark 10. [Removing fully covered regions] If full $(k)=1$, as the whole region is proved to belong to the DA of the origin, such a region can be actually removed from $\Omega^{[c]}$ in step 2 of Algorithm 1; in order to keep this information, the faces of neighboring regions can be "marked" to belong to the DA via suitable set up of ellipsoids $\hat{\mathcal{E}}_{k}$.

Remark 11. [Geometric optimisation goal] In general, there are no LMI conditions to maximise the volume of a piecewise estimation of the DA. An indirect 
Algorithm 1. Start from a compact set $\Omega^{[0]}$ defined by a list of sets from a associated partition $\Omega_{k}, k \in\{1,2, \ldots, q\}$. Consider a previous estimate of the DA, see Remark 8 , as a list of sets in the form $\mathcal{E}_{k}^{[0]}=\left\{x: \bar{x}^{T} \bar{G}_{k}^{0} \bar{x}>0\right\} \cap \Omega_{k}$. Set $c=1$ and perform the following steps:

1. Test Lemma 4 for each region $\Omega_{k} \in \Omega^{[c-1]}$.

(a) If a) is feasible, set full $(k)=1$ else full $(k)=0$.

(b) If b) is feasible, set empty $(k)=1$ else $\operatorname{empty}(k)=0$.

2. Generate the list of sets for a new partition $\Omega^{[c]}$, as follows:

(a) If empty $(k)=1$, then reject $\Omega_{k}$, do not add it to $\Omega^{[c]}$;

(b) Else, add $\Omega_{k}$ to the list $\Omega^{[c]}$, and enlarge the region of study adding to $\Omega^{[c]}$ a neighbouring region $\Omega^{\prime}$, see Remark 9 .

(c) if $\operatorname{full}(k)=1, \Omega_{k}$ can be taken out, if so wished, from $\Omega^{[c]}$, if the steps in Remark 10 are taken.

3. Obtain a new PWATS model from the new region.

4. Obtain a PWQLF from Theorem 4 under some chosen geometric performance maximisation, see Remark 11.

5. If Theorem 4 is feasible, then add $\left\{x: \bar{x}^{T} \bar{P}_{k}^{c} \bar{x}<0\right\} \cap \Omega_{k}$ to the list of sets conforming the current DA estimate, and set $c=c+1$.

6. If Theorem 4 is not feasible, then subdivide some of the regions where empty $(k)=0$ and $\operatorname{full}(k)=0$. See Remark 12 .

7. Check a suitable termination criteria (see Remark 13), and if it not satisfied, go to Step 1.

way to achieve this goal is to maximise the radius of a sphere centered at the origin [10], but it may be inadequate for nonconvex regions. An alternative to the sphere-based maximisation is trying to maximise in a region the scaling (29) of a degenerate ellipsoid (with very small axis length in all directions but a random one) with a random center point. 
Remark 12. [Finer partition granularity] As expected, there are several ways of dividing regions as to apply the algorithm above; in later examples in this work, the regions have been split into $2^{n}$ equal smaller parallelotopes. Obviously, other implementations may be conceivable, such as generating a random splitting direction for some regions.

Remark 13. [Termination] There might be different options to be used as termination criteria: (a) some geometric goal reached, or slow progress of it, (b) number of regions or computation time at step 4 above a predefined limit.

\section{Comparative analysis with other DA analysis proposals}

In [20], an algorithm to get progressively better estimates of the DA was given. Nevertheless, in contrast with Algorithm 1 above, the proposal in [20] (a) is unable to establish asymptotical exactness (see next section); (b) it includes no geometrical optimisation conditions, thus stopping when any arbitrary piecewise Lyapunov set which fits the DA is found; (c) it is computationally over-demanding since at each step the whole region is reconsidered in the new partition. All these issues make the prior algorithm provide worse numerical results than the one here presented (see example below).

Example 2. Consider the following nonlinear system

$$
\dot{x}_{1}=-x_{2}, \quad \dot{x}_{2}=x_{1}-x_{2}+x_{2} x_{1}^{2} .
$$

The system has one equilibrium point at the origin and one unstable limit cycle, which implies the DA is bounded by the latter. In order to obtain the largest possible estimate of the DA, Algorithm 1 comes at hand. We started it with the region $\Omega^{[0]}=\left\{x \in \mathbb{R}^{2}:\left|x_{i}\right| \leq 0.99\right\}, i \in\{1,2\}$, on which a quadratic Lyapunov function has been used as an initial estimate of the DA.

Figure 5 plots the limit cycle (outermost blue closed curve, obtained with backwards-in-time simulation) and compares it with different estimates of the DA obtained by the iterations of Algorithm 1. The figure shows, in different colors, the estimate of DA for each iteration of Algorithm 1. Note that, in this example, the chosen geometry partition is based on a square tessellation, 
and we maximised the radius of a sphere center at the origin as the geometric optimisation goal. A colored square means that the entire region belongs to the DA. The different sizes of the regions are caused by the splitting into smaller squares at step 6 of the Algorithm. The region proven to belong to the DA is the union of all colored regions.

Figure 6 shows the DA estimate in Figure 5 as a red line, very close to the actual exact limit cycle (black line). For comparison, it also shows the result applying the approach in [20] with a blue closed solid line. The approach in [20] does not incorporate the geometric border conditions neither previous estimates, reaching a high computational cost with slow progress, obtaining inferior results. Both algorithms were stopped when 4 GB of memory were exhausted in the computations.

As the algorithm progresses, it gets progressively closer to the actual domain of attraction of the origin (the open set inside the limit circle). However, as the boundary of the limit cycle is not quadratic, we would, in theory, need an infinite amount of piecewise-quadratic fragments to approximate it, this is why the number of regions ends up increasing greatly.

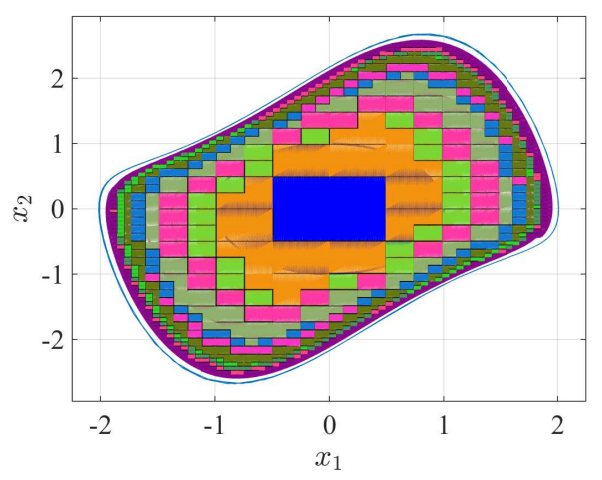

Figure 5: Estimation of the DA for Example 2.

Next section analysis in depth the algorithm behaviour when the number of regions increases: it can be proved that, under some assumptions, as the partitions get finer, the accuracy of the DA estimate improves, reaching asymp- 


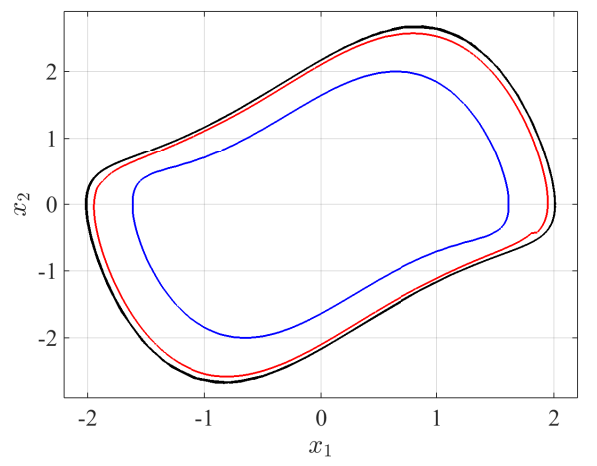

Figure 6: Estimation of the DA for Example 2 (Black: exact Limit Cycle; red: proposal here; blue: estimate in [20]).

totical exactness i.e., limited only by finite computational resources in DA estimation (disturbances and controller design induce other limitations as more complex/BMI problems arise, out of the scope of this work).

\section{Asymptotical exactness}

In this section, Farkas Lemma (here recalled as Lemma 1) will allow to prove asymptotical exactness of the above algorithm: with enough computational resources, the algorithm is non-conservative in the precise sense to be discussed next.

Indeed, Theorems 3 and 4, obviously, apply to the particular case in which the Lyapunov function has the form

$$
V_{k}(x)=\bar{x}^{T}\left[\begin{array}{cccc}
0 & 0 & \cdots & 0.5 p_{k}^{1} \\
0 & 0 & \cdots & 0.5 p_{k}^{2} \\
\vdots & \vdots & \ddots & \vdots \\
0.5 p_{k}^{1} & 0.5 p_{k}^{2} & \cdots & p_{k}^{n+1}
\end{array}\right] \bar{x}=\bar{x}^{T} \bar{P}_{k} \bar{x}
$$

These Lyapunov functions are piecewise-affine, as $V_{k}(x)=\sum_{i=1}^{n} p_{k}^{i} x_{i}+p_{k}^{n+1}$, shorthanded to PWALF. In this way, piecewise-polyhedral level sets could be proven to belong to the DA of the origin. 
The key fact about the use of the above class of functions is that, due to Lemma 1, the proposed conditions in Theorem 3 are necessary and sufficient in the sense that, if conditions in the referred theorem with the above Lyapunov function structure (33) are not feasible then there is no PWALF for the set partition fulfilling the needed Lyapunov condition ${ }^{10}$ with a single affine expression for the PWALF in each $\Omega_{k}$. So, forcedly, the partition must be changed, because no other theorem would find a PWALF on it if Theorem 3 does not work.

The above idea, jointly with universal-approximation capabilities of PWALF and PWATS models as regions get smaller, allow to prove the following key result, which states that if there exists any smooth Lyapunov function proving that a particular point $x^{*}$ belongs to the DA of the origin, a PWALF will also prove that $x^{*}$ belongs to such DA for a fine enough partition.

Lemma 5. For any $\varepsilon_{1}>0, \varepsilon_{2}>0$, there exist a fine enough partition of a compact set $\Omega$ such that a PWALF in the form (33), $V_{P W}(x):=V_{k}(x)$ for $x \in \Omega_{k}$, approximates any function $V$ of class $\mathscr{C}^{2}$ and its gradient as follows, for all $x \in \Omega$ :

$$
\begin{gathered}
\left\|V_{P W}(x)-V(x)\right\| \leq \varepsilon_{1}, \\
\left\|\nabla V_{P W}(x)-\nabla V(x)\right\| \leq \varepsilon_{2} .
\end{gathered}
$$

Proof. See Appendix.

Lemma 6 . For any $\varepsilon>0$, there exist a fine enough partition of a compact set $\Omega$ such that, given a continuous function $f(x)$, a PWATS model can be obtained

\footnotetext{
${ }^{10}$ Contrarily, in the quadratic case, such a Lyapunov function might exist but might be only provable to be so with higher-degree Positivstellensatz multipliers, requiring a Sum-of-Squares version of the theorems; anyway, there are also positive polynomials which are not SOS [25] so these conservatism sources cannot be removed in general, except in the above-referred affine case.
} 
fulfilling:

$$
\begin{aligned}
& \left\|\left(A_{i}^{k} x+b_{i}^{k}\right)-f(x)\right\| \leq \varepsilon, \\
& \forall i \in\{1,2, \ldots, r\}, \forall x \in \Omega_{k}
\end{aligned}
$$

Proof. Consider the 2-rule PWATS model given by $A_{i}^{k}:=0, b_{1}^{k}:=\min _{x \in \Omega_{k}} f(x)$, $b_{2}^{k}:=\max _{x \in \Omega_{k}} f(x)$, where maximum and minimum have been considered to be computed element-wise ( $b_{1}^{k}$ and $b_{2}^{k}$ are vectors) on a compact set $\Omega_{k}$. As $f(x)$ is continuous, by assumption, there exists a fine enough partition such that $\left\|b_{i}^{k}-f(x)\right\| \leq\left\|b_{2}^{k}-b_{1}^{l}\right\| \leq \varepsilon$ for any arbitrary choice of $\varepsilon$.

Now, we can state the key result of this paper, proving that we can be at least as good as any conceivable algorithm based on Lyapunov level-sets.

Theorem 5. Let $x=0$ be an asymptotically stable equilibrium point for the nonlinear system

$$
\dot{x}=f(x)
$$

where $f: \Omega \rightarrow \mathbb{R}^{n}$ is locally Lipschitz, $\Omega \subset \mathcal{D}$ is compact. Assume that a (possibly small) polyhedron $\mathcal{B}$ containing the origin has been proved to belong to the $D A$, and define a compact set $\Theta:=\Omega-i n t(\mathcal{B})$. If there exists a function $V: \Theta \rightarrow \mathbb{R}$, and $\varepsilon>0$ such that:

1. $V(x)$ is of class $\mathscr{C}^{2}$ in an open set including $\Omega$.

2. $\dot{V}(x)=\frac{\partial V}{\partial x} \cdot f(x) \leq-\varepsilon$, for all $x \in \Theta$.

3. There exists a level set in the form $V_{\alpha_{2}}:=\left\{x: V(x) \leq \alpha_{2}\right\}$, for some $\alpha_{2}>0$ such that $V_{\alpha_{2}} \subset \Omega$.

Then, there exist a fine enough partition of $\Theta$ such that any PWATS model fulfilling conditions in Lemma 6 allows finding a PWQLF $\left(V_{P W}(x)\right)$ which fulfills conditions in Theorem 3, and a level set of the PWQLF allowing to prove that any point in the interior of $V_{\alpha_{2}}$ belongs to the DA of the origin.

Proof. See Appendix. 


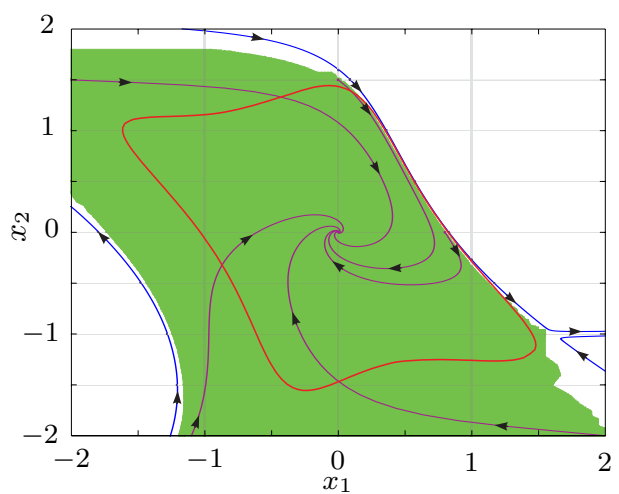

Figure 7: Estimation of the DA for Example 3. (Red: estimate in [14]; Green: proposal here; magenta: some trajectories inside the DA; blue: some trajectories outside DA.

Remark 14. Note that, by Theorem 1 , all trajectories of the nonlinear system inside the level set $V_{\alpha_{2}}$ will enter $\mathcal{B}$, because forcedly $V_{\alpha_{2}} \cap \mathcal{B} \neq \emptyset$, as the trajectories should abandon $V_{\alpha_{2}}$ in at most $\alpha_{2} / \varepsilon$ time units, and they cannot abandon $\Omega$ if they start in the interior of $V_{\alpha_{2}}$. For any of such interior initial conditions, a PWQLF proving that it belongs to the DA of the origin can be found because of the same argumentations.

Example 3. As a last example, for the sake of comparison, consider the system in [14, Example 3]:

$$
\dot{x}_{1}=-x_{1}+x_{1}^{2}+x_{1}^{3}+x_{1}^{2} x_{2}-x_{1} x_{2}^{2}+x_{2}, \quad \dot{x}_{2}=-\sin x_{1}-x_{2},
$$

altogether with a PWATS model of it, [10], as an input to Algorithm 1. Figure 7 shows the DA estimate in the referred work (obtained via BMIs and SOS tools) with a red closed solid line whereas our estimate is shown with a greencoloured area. Clearly, our proposal reaches much better estimations than [14], as expected due to the asymptotical exactness; however, region size needs to be decreased as the border of the "true" domain of attraction is approached, as discussed in earlier examples.

Remark 15. With prefixed regions, our proposal renders LMI conditions (even linear programming ones, in some cases) so the computational cost is basically 
identical to prior PWATS literature (increasing just a small amount due to the handful of extra multipliers proposed here). However, the actual DA of nonlinear systems is, in general, not piecewise quadratic, so the exact domain of attraction cannot be obtained with finite computational resources with our ap$\operatorname{proach}^{11} .:$ as the required estimation accuracy increases, the number of regions must increase (with decreasing size). Hence, Theorem 5 can only prove that finite computational resources are needed to find a particular point in the interior of the "true" DA.

\section{Conclusion}

In this paper, an iterative linear matrix inequality methodology has been presented for estimation of the domain of attraction of a nonlinear model. The proposal, based on a systematic exploitation of geometrical and stability facts via piecewise affine Takagi-Sugeno models and piecewise Lyapunov functions, has been shown to outperform the most relevant works on the subject. Estimates of the domain of attraction have been increased by "emptying" previously proven regions and extending the modelling region in "promising" neighboring areas. Moreover, based on universal-approximation properties of TS models, it has been proved that the estimate of the domain of attraction approaches the level set of any existing $\mathscr{C}^{2}$ Lyapunov function of the original nonlinear system, as the partition where the piecewise TS model is obtained gets finer (smaller regions): the proposed procedures are asymptotically exact.

\section{Appendix A. Proofs}

Proof of Corollary 1. The result can be proved considering $V(x)=0$ as $V(x) \geq$ $0,-V(x) \geq 0$, and applying twice the above lemma, i.e., for $V(x)=0, \forall x \in \Omega$,

\footnotetext{
${ }^{11}$ In fact, neither with any alternative conceivable approach: it is well known that nonlinear differential equations rarely admit explicit solutions (or DA expressions) in closed form, requiring numerical simulation [29]
} 
there exist $c_{1} \geq 0$ and $c_{2} \geq 0$ such that:

$$
V(x)-\sum_{l=1}^{N} \tau_{l}^{\prime} \sigma_{l}(x)=c_{1} \geq 0,-V(x)-\sum_{l=1}^{N} \tau_{l}^{*} \sigma_{l}(x)=c_{2} \geq 0
$$

where linearity of $V$ forces $c_{1}$ and $c_{2}$ being constants. Adding, we would have:

$$
-\sum_{l=1}^{N}\left(\tau_{l}^{\prime}+\tau_{l}^{*}\right) \sigma_{l}=c_{1}+c_{2}
$$

but, if we assume the region $\Omega$ is not empty, the above cannot happen unless $c_{1}=c_{2}=0$ (standard Positivstellensatz). Now, subtracting and dividing by 2 , we obtain:

$$
V-\sum_{l=1}^{N} \frac{1}{2}\left(\tau_{l}^{\prime}-\tau_{l}^{*}\right) \sigma_{l}=0
$$

so $\tau_{l}=0.5\left(\tau_{l}^{\prime}-\tau_{l}^{*}\right)$.

Proof of Theorem 3. Consider the regions $\hat{\mathcal{E}}_{k}:=\bigcup_{j=1}^{n_{k}}\left(\bigcup_{s=1}^{\hat{s}_{k}} \hat{\mathcal{E}}_{j s}^{k} \cap \mathcal{F}_{j}^{k}\right)$ and $\mathcal{E}_{k}:=\Omega_{k} \cap \bigcup_{s=1}^{\bar{s}_{k}} \mathcal{E}_{s}^{k}$. Consider, too, the regions $\mathcal{E}:=\bigcup_{k=1}^{q} \mathcal{E}_{k}$ and $\hat{\mathcal{E}}:=\bigcup_{k=1}^{q} \hat{\mathcal{E}}_{k}$. Then, by assumption, each $\mathcal{E}_{k}, \hat{\mathcal{E}}_{k}$, and, evidently, the whole $\hat{\mathcal{E}}$, and $\mathcal{E}$ belong to the DA of the origin.

Using the argumentations in Lemma 3 with $\Xi=\dot{V}_{k}(x)+\gamma\|x\|^{2}$ and $V_{k}(x):=$ $\bar{x}^{T} \bar{P}_{k} \bar{x}$, we can state that (25) ensures that the time derivative of $V_{k}(x)$ is strictly negative for nonzero $x$ (lower or equal than $-\gamma\|x\|^{2}$ ), in $\Omega_{k}-\mathcal{E}_{k}$, because such set is given by:

$$
\Omega_{k}-\mathcal{E}_{k}=\left\{x: E_{k} \bar{x} \succeq 0, \bar{x}^{T} Q_{l k} \bar{x} \geq 0, \bar{x}^{T} \bar{G}_{s k} \bar{x} \leq 0\right\}
$$

for $l \in\left\{1,2, \ldots, \ell_{k}\right\}$ and $s \in\left\{1,2, \ldots, \bar{s}_{k}\right\}$, so suitable multipliers $U_{k i}^{1} \succeq 0$, $\tau_{k l}^{1} \geq 0, \tau_{k s}^{2} \geq 0$ are introduced.

Let us discuss now inequality (26). In this case, we want to show that the level set $\left\{V_{k}(x)<0\right\} \cap\left(\Omega_{k}-\mathcal{E}_{k}-\hat{\mathcal{E}}_{k}\right)$ does not intersect $\partial \Omega^{\uparrow}$, as $\partial \Omega^{\uparrow}$ is the subset of $\partial \Omega_{k}$ where the trajectories of the system do not immediately enter $\Omega$.

In order to show that, we will combine Corollary 4 with Lemma 3, posing the conditions of $\bar{P} \geq 0$ for all $x$ in the set $\partial \Omega^{\uparrow} \cap\left(\Omega_{k}-\mathcal{E}_{k}-\hat{\mathcal{E}}_{k}\right)$. 
As $\partial \Omega^{\uparrow} \subset \bigcup_{j \in \mathcal{I}_{k}}\left\{x: \dot{\sigma}_{j}^{k}(x) \leq 0\right\}$ we can assert that, if the following assertion holds for all $j \in \mathcal{I}_{k}$ :

$$
\bar{P} \geq 0 \forall x \in \Sigma_{j}^{k}
$$

where $\Sigma_{j}^{k}:=\left\{x: \dot{\sigma}_{j}^{k}(x) \leq 0\right\} \cap\left(\Omega_{k}-\mathcal{E}_{k}-\hat{\mathcal{E}}_{k}\right)$, then $\bar{P} \geq 0$ on $\partial \Omega^{\uparrow} \cap\left(\Omega_{k}-\mathcal{E}_{k}-\hat{\mathcal{E}}_{k}\right)$.

Now, we replace $\Sigma_{j}^{k}$ by the larger (shape-independent) set on which at least one of the vertices of the PWATS model proves $\dot{\sigma}_{j}^{k}(x) \leq 0$, as discussed on Corollary 4. Then, application of Lemma 3 for each of the outer constraints in (A.1) and model vertices yields conditions (26) if the constraint in consideration is affine, and (28) if it were quadratic.

Now, by considering all regions we have:

1. a continuous piecewise quadratic function $V(x)$, defined as $V_{k}(x)=\bar{x}^{T} \bar{P}_{k} \bar{x}$ in $\Omega_{k}$;

2. $V(x)$ is non-increasing, i.e., for a sufficiently small $\epsilon, V(x(t+\epsilon)) \leq V(x(t))$; actually $V(x(t+\epsilon))<V(x(t))$ if $x(t) \neq 0$. Indeed, along the trajectories of the nonlinear system (1), $\dot{V} \leq 0$ if $x(t)$ is in the interior of any $\Omega_{k}$; if $x(t)$ is in the boundary of several regions, we can ensure that:

$$
D^{+} V(t):=\lim _{\epsilon \rightarrow 0^{+}} \frac{V(x(t+\epsilon))-V(x(t))}{\epsilon} \leq \max _{k \text { s.t. } x(t) \in \Omega_{k}} \dot{\dot{V}}_{k} \leq 0
$$

3. $V(x)$ has a level-zero set $V_{0}:=\{V(x)<0\}$ that verifies

$$
V_{0} \cap\left(\partial \Omega^{\uparrow} \cap\left(\Omega_{k}-\mathcal{E}_{k}-\hat{\mathcal{E}}_{k}\right)\right)=\emptyset .
$$

Denoting $\mathcal{E}:=\mathcal{E} \cup \hat{\mathcal{E}}$, Let us define the following sets:

$$
\begin{gathered}
\mathcal{V}:=\{V(x)<0\} \cap \Omega, \quad \mathcal{W}:=\mathcal{V}-\mathcal{E}, \\
\mathcal{W}_{\neg \varepsilon}:=\{x \in \mathcal{W}: \phi(t, x) \notin \mathcal{E} \forall t \geq 0\}, \mathcal{W}_{\varepsilon}:=\mathcal{W}-\mathcal{W}_{\neg \varepsilon} .
\end{gathered}
$$

With the above definition, $\mathcal{W}$ is the set of points who have not (yet) been proven to belong to the DA. Such set is partitioned in two: $\mathcal{W}_{\neg \varepsilon}$, i.e., the set of points of $\mathcal{W}$ which do not enter $\mathcal{E}$ in finite time, and $\mathcal{W}_{\boldsymbol{\varepsilon}}$.

Now, note that when starting in $\mathcal{W}$, it is impossible to abandon $\mathcal{W}$ without entering $\mathcal{E}$, due to: 
- As $V(x)$ is non-increasing in time in $\mathcal{W}$, the boundary $V(x)=0$ will never be reached.

- As $\dot{\sigma}_{j}^{k}(x)>0$ for all $x$ lying both in the outer faces and in $V(x)<0$ (proven due to the third of the above-enumerated conditions), trajectories cannot exit $\Omega$ through such outer faces.

Thus, all points in $\mathcal{W}$ either enter $\mathcal{E}$ in finite time or remain indefinitely in $\mathcal{W}$. As the latter points are, by definition, those in $\mathcal{W}_{\neg \varepsilon}$, forcedly $\mathcal{W}_{\varepsilon}$ is the set of points who do enter $\mathcal{E}$ in finite time.

Obviously, all $x \in \mathcal{W}_{\varepsilon}$ belong to the DA of the origin, because they enter $\mathcal{E}$ in finite time without leaving $\Omega$, so they converge to the origin later on.

Let us prove that all $x \in \mathcal{W}_{\neg \varepsilon}$ belong, too, to the DA of the origin. Indeed, $\mathcal{W}_{\neg \varepsilon}$ is invariant, because trajectories always remain inside it in future time: they do not enter $\mathcal{E}$ and, due to the above reasons, they do not exit $\mathcal{V}$, and they do not enter $\mathcal{W}_{\boldsymbol{\varepsilon}}$ because in such a case they would eventually enter $\mathcal{E}$, which cannot happen by definition.

As $V(x)$ is continuous, piecewise polynomial, it is bounded on $\mathcal{W}_{\neg \varepsilon}$, i.e., there exist

$$
V_{\min }:=\inf _{x \in \mathcal{W}_{\neg \varepsilon}} V(x), \quad V_{\max }:=\sup _{x \in \mathcal{W}_{\neg \varepsilon}} V(x) .
$$

Given any $x \in \mathcal{W}_{\neg \varepsilon}$, as $V(\phi(t, x))$ is nonincreasing and bounded from below at all times, there must exist a limit $a:=\lim _{t \rightarrow \infty} V(\phi(t, x))$, so, as a consequence $\lim _{t \rightarrow \infty} D^{+} V(\phi(t, x))=0$. As $\dot{V}_{k}(x) \leq-\gamma$ in regions $\Omega_{k}$ not containing the origin, and $\dot{V}_{k}(x) \leq-\gamma\|x\|^{2}$ if the region contains the origin, the only point in which such situation $\left(D^{+} V=\max _{k}\right.$ s.t. $\left.x(t) \in \Omega_{k} \dot{V}_{k}=0\right)$ can happen is the origin. So, all initial conditions $x \in \mathcal{W}_{\neg \varepsilon}$ tend to the origin, i.e., belong to the DA of the origin ${ }^{12}$. Given that both $\mathcal{W}_{\varepsilon}$ and $\mathcal{W}_{\neg \varepsilon}$ belong to the DA of the origin, so does their union $\mathcal{W}$.

Proof of Theorem 4. In this case, we want to show that $\hat{\Omega}(\lambda)$ belongs to the

\footnotetext{
${ }^{12}$ Note that, if $0 \in \mathcal{E}$, forcefully $\mathcal{W}_{\neg \varepsilon}=\emptyset$; this is in accordance with Theorem 1 .
} 
domain of attraction of $x=0$, by showing that it is included in the subset of the DA proven in Theorem 3, where constraints for the level set $\mathcal{V}$ for being part of the DA are enforced ((24), (25), (26), (28)).

We want to enforce that the region $\mathcal{E}_{k^{\prime}} \cup\left\{\bar{x}^{T} \bar{P}_{k^{\prime}} \bar{x}<0\right\} \cap \Omega_{k}$ contains $\hat{\Omega}(\lambda) \cap$ $\Omega_{k^{\prime}}$. We will do that by proving that $\bar{x}^{T} \bar{P}_{k^{\prime}} \bar{x} \leq-\gamma$ in $\left(\Omega_{k^{\prime}}-\mathcal{E}_{k^{\prime}}\right) \cap \hat{\Omega}(\lambda)$. Indeed, if that holds, all points of $\hat{\Omega}(\lambda) \cap \Omega_{k^{\prime}}$ either lie in $\mathcal{E}_{k^{\prime}}$ or in $\left\{\bar{x}^{T} \bar{P}_{k^{\prime}} \bar{x}<0\right\} \cap \Omega_{k}$, both belonging to the DA of the origin.

Thus, conditions for inclusion of $\bar{x}^{T} \bar{P}_{k^{\prime}} \bar{x} \leq-\gamma$ in the required set are written as (30) by using the S-procedure argumentation and positive multipliers $\tau_{k^{\prime} m}^{8}$ associated to the quadratic constraints in $\hat{\Omega}(\lambda), U_{k^{\prime}}^{4}$ associated to the linear inequalities in $\hat{\Omega}(\lambda), U_{k^{\prime}}^{3}$ and $\tau_{k^{\prime} l}^{7}$ associated to the corresponding region $\Omega_{k}$, and positive constants $\tau_{k^{\prime} s}^{9}$ associated to ellipsoids $\mathcal{E}_{s}^{k}$.

Proof of Corollary 6. Suppose that a feasible solution $\left\{P_{k}^{J o h}, U_{k}^{J o h}, W_{k i}^{J o h}\right\}$ for (15) has been obtained, i.e.:

$$
\begin{gathered}
\bar{P}_{k}^{J o h}-\bar{E}_{k}^{T} U_{k}^{J o h} \bar{E}_{k} \geq \mathbf{I}_{\gamma}, \\
\left(\bar{A}_{i}^{k}\right)^{T} \bar{P}_{k}^{J o h}+\bar{P}_{k}^{J o h} \bar{A}_{i}^{k}+\bar{E}_{k}^{T} W_{k i}^{J o h} \bar{E}_{k} \leq-\Phi_{\gamma}^{k} .
\end{gathered}
$$

We will prove that there exist some $\beta>0$ such that $V_{\beta}$ in Theorem 2 belongs to the DA of the origin, provable with Theorem 3. As the level set considered in the latter theorem is in the form $\left\{\bar{x}^{T} \bar{P}_{k} \bar{x}<0\right\}$, whereas the condition $x^{T} \bar{P}_{k}^{J o h} \bar{x} \geq$ $I_{\gamma}$ in Theorem 2 would need level sets in the form $\left\{\bar{x}^{T} \bar{P}_{k}^{J o h} \bar{x}<\beta\right\}$, we will consider $\bar{P}_{k}=\bar{P}_{k}^{J o h}-\mathbf{0}_{\beta}$, without loss of generality, for some $\beta$. In this way, $\left\{\bar{x}^{T} \bar{P}_{k}^{J o h} \bar{x}<\beta\right\} \equiv\left\{\bar{x}^{T} \bar{P}_{k} \bar{x}<0\right\}$.

Consider inequality (25). As partition is polyhedral then $\ell_{k}=0$ and if the prior estimates of the DA are empty, then $\bar{s}_{k}=0$ and $\hat{s}_{j k}=0$. Furthermore if only the rows $E_{k}$ are considered from $\bar{E}_{k}$, the result is the second LMI in (A.5), with the notational changes in footnote 4 . As subtracting a constant from the Lyapunov function does not influence its derivative (algebraically, it can be proved from the fact that the last row of $\bar{A}_{i}^{k}$ is zero), Johansson's multipliers $W_{k i}^{J o h}$ would render (25) feasible (padded with zeros to conform the larger size of $\left.\bar{E}_{k}\right)$. 
Consider now that the first inequality in (A.5) holds. Then, we will prove that there exists $\beta>0$ and arbitrary row-vector multipliers $Z_{j k}$ such that

$$
Z_{j k}^{T} E_{j k}+(*)+\left(\bar{P}_{k}^{J o h}-\mathbf{0}_{\beta}\right)-E_{k}^{T} U_{k}^{J o h} E_{k} \geq 0
$$

where the above expression has been obtained from (26) removing the absent elements $Q_{l k}, \bar{G}_{s k}, \hat{G}_{k j s}$, and also setting the multiplier for the term $E_{j k} \bar{A}_{i}^{k}$ in $U_{k j i}^{2}$ equal to zero (hence, the original multiplier $U_{k j i}^{2}$ no longer depends on $i$, $j$ ), setting the remaining terms equal to the corresponding ones in $U_{k}^{J o h}$.

Indeed, consider the problem of finding $E_{j k}$ such that the following expression is feasible for all outer constraints $E_{j k}$ :

$$
Z_{j k}^{T} E_{j k}+(*)+\operatorname{blkdiag}(\gamma I,-\beta) \geq 0
$$

The above problem is feasible if the circle $\gamma x^{T} x \leq \beta$ is inside $\Omega$. So, if there exists a circle around the origin which is contained in $\Omega$, true by assumption, a feasible solution for (A.7) exists. Now, adding the first matrix inequality of (A.5) and (A.7) results in (A.6), proving that (26) was feasible in Theorem 3 with the choice of multipliers in (A.6).

In summary, the above argumentation proves that if (A.5) are feasible, so they are (25) and (26). Continuity is also enforced in Johansson's result, so we proved that Theorem 3 is feasible in all cases (A.5) is, for suitable $\Omega$.

Proof of Lemma 5. First, note that the gradient of a PWALF is a piecewiseconstant function ${ }^{13}$. If a function $V(x)$ is of class $\mathscr{C}^{2}$, then its partial derivative $\nabla V$ is of class $\mathscr{C}^{1}$, meaning that $\nabla V$ is bounded in $\Omega$ and can be approximated by a piecewise constant function $\nabla V_{P W}$ to any arbitrary error $\varepsilon_{3}$, as piecewise constant functions are universal function approximators, as long as the partition is fine enough, so there exists $\psi(x)$ such that $\|\psi(x)\| \leq \varepsilon_{3}$ for all $x \in \Omega$ and $\nabla V(x)=\nabla V_{P W}(x)+\psi(x)$.

\footnotetext{
${ }^{13}$ Understanding the gradient at faces common to several regions to be defined as the average of the different piecewise gradients. As such faces are zero-measure sets, such formal definition will not have any influence in the integral-based results in the remaining of the proof.
} 
Integrating the gradient, we get:

$$
V(x)=\int_{0}^{1} \nabla V(\lambda x)^{T} x d \lambda=\int_{0}^{1}\left(\nabla V_{P W}(\lambda x)+\psi(\lambda x)\right)^{T} x d \lambda
$$

where $\psi(\lambda x)$ is the approximation error, which verifies $\|\psi(\lambda x)\| \leq \varepsilon_{3}$. Hence,

$$
V(x)=\int_{0}^{1} \nabla V_{P W}(\lambda x)^{T} x d \lambda+\int_{0}^{1} \psi(\lambda x)^{T} x d \lambda
$$

so we can assert:

$$
\left\|V(x)-V_{P W}(x)\right\| \leq \int_{0}^{1}\|\psi(\lambda x)\| \cdot\|x\| d \lambda \leq \varepsilon_{3}\|x\|
$$

Choosing $\varepsilon_{3}$ such that $\varepsilon_{1} \geq \max _{x \in \Omega} \varepsilon_{3}\|x\|$, and $\varepsilon_{3} \leq \varepsilon_{2}$, we can prove (34) and (35). As a result, we can approximate both $\nabla V$ and $V$ as closely as desired by increasing the partition granularity.

Proof of Theorem 5. By Lemma 5, there exists a fine enough partition such that there exists a PWA function fulfilling: $\nabla V_{P W}(x)+\psi(x)=\nabla V(x),\|\psi(x)\| \leq \varepsilon_{3}$, and, by Lemma 6 , that for all vertices, for all regions there exists $\phi_{i}^{k}(x)$ such that $A_{i}^{k} x+b_{i}^{k}+\phi_{i}^{k}=f(x),\left\|\phi_{i}^{k}(x)\right\| \leq \varepsilon_{4}$, for any $\varepsilon_{4}>0$. Then, we can state, denoting $f_{i}^{k}(x):=A_{i}^{k} x+b_{i}^{k}$, by continuity of $f(x)$ that there exists $\hat{f}:=\max _{x \in \Omega}\|f(x)\|$, and by continuity of $\nabla V$, that there exists $\hat{V}:=\max _{x \in \Omega}\|\nabla V(x)\|$. Now, we have:

$$
\begin{aligned}
& \nabla V_{P W}(x) f_{i}^{k}(x)=(\nabla V-\psi(x))\left(f(x)-\phi_{i}^{k}(x)\right) \\
& =\nabla V \cdot f(x)-\psi(x) \cdot f(x)-\nabla V \cdot \phi_{i}^{k}(x)+\psi(x) \phi_{i}^{k}(x) \\
& \quad \leq-\varepsilon+\varepsilon_{2} \cdot \hat{f}+\varepsilon_{4} \cdot \hat{V}+\varepsilon_{4} \varepsilon_{2}
\end{aligned}
$$

So, for any $0<\gamma^{\prime}<\varepsilon$, a suitable choice of small enough $\varepsilon_{2}$ and $\varepsilon_{4}$ can prove that there exists a fine enough partition so that:

$$
\nabla V_{P W}(x) f_{i}^{k}(x) \leq-\gamma^{\prime}
$$

Now, from Farkas Lemma, the existence of the multipliers $U_{k i}^{1}$ in $(25)$ in the affine case $\left(l_{k}=0, \bar{s}_{k}=0\right)$ are a necessary and sufficient condition for (A.11) to hold, as the region $\Omega_{k}$ does not contain the origin by assumption. Regarding 
the multiplier-based continuity conditions (19), Corollary 1 ensures that they are also necessary and sufficient for the PWA case.

Last, regarding geometric conditions (level set), any point in the interior of $V_{\alpha_{2}}$ is in the (closed) level set $\alpha_{1}$ for some $\alpha_{1}<\alpha_{2}$.

Consider now $\varepsilon_{1}<0.5\left(\alpha_{2}-\alpha_{1}\right)$. Then, select any choice of $\alpha$ such that $\alpha_{1}+\varepsilon_{1}<\alpha<\alpha_{2}-\varepsilon_{1}$. In this way, given the above $\varepsilon_{1}$, there exists a fine enough partition so that (34) holds; hence, the level set of $V_{P W}$, denoted as $\tilde{V}_{P W}(\alpha):=\left\{x: V_{P W}(x) \leq \alpha\right\}$, fulfills

$$
V_{\alpha_{1}} \subset \tilde{V}_{P W}(\alpha) \subset V_{\alpha_{2}}
$$

because all $x \in V_{\alpha_{1}}$ will belong to the level set of $V_{P W}$ given by $\tilde{V}_{P W}\left(\alpha_{1}+\varepsilon_{1}\right)$, and also, all elements of the level set $\tilde{V}_{P W}\left(\alpha_{2}-\varepsilon_{1}\right)$ will be included in $V_{\alpha_{2}}$.

If a fine enough partition is chosen such that both (A.11) and (A.12) hold, we have found a PWALF fulfilling the required derivative conditions and including in a level set any desired point in the interior of the level set of the "true" Lyapunov function. If we consider that piecewise-affine Lyapunov functions are a particular case of piecewise-quadratic ones, the theorem is proved.

\section{Acknowledgment}

The authors gratefully acknowledge the support of the following institutions: Project Ciencia Básica SEP-CONACYT CB-168406, the CONACyT/COECYT Sonora scholarship 383252, project DPI2011-27845-C02-01 (Spanish government, MINECO), and the scholarship GRISOLIA/2014/006.

\section{References}

[1] T. Taniguchi, K. Tanaka, H. Wang, Model construction, rule reduction and robust compensation for generalized form of Takagi-Sugeno fuzzy systems, IEEE Transactions on Fuzzy Systems 9 (2) (2001) 525-537. 
[2] H. Wang, K. Tanaka, M. Griffin, An approach to fuzzy control of nonlinear systems: Stability and design issues, IEEE Transactions on Fuzzy Systems 4 (1996) 14-23.

[3] S. Boyd, L. Ghaoui, E. Feron, V. Belakrishnan, Linear Matrix Inequalities in System and Control Theory, Vol. 15, SIAM: Studies In Applied Mathematics, Philadelphia, USA, 1994.

[4] J. Sturm, Using SeDuMi 1.02, a MATLAB toolbox for optimization over symmetric cones, Optimization Methods and Software 11 (1-4) (1999) 625653.

[5] F. Wu, H. Li, $H_{\infty}$ fuzzy observer-based control for a class of nonlinear distributed parameter systems with control constraints, IEEE Transactions on Fuzzy Systems 16 (2) (2008) 502-516.

[6] J. Qiu, G. Feng, H. Gao, Fuzzy-model-based reliable static output feedback $H_{\infty}$ control of nonlinear hyperbolic PDE systems, IEEE Transactions on Fuzzy Systems 24 (2) (2016) 388-400.

[7] A. Sala, On the conservativeness of fuzzy and fuzzy-polynomial control of nonlinear systems, Annual Reviews in Control 33 (1) (2009) 48-58.

[8] J. Chen, S. Xu, Y. Li, Q. Z., Y. Chu, Improvement on stability conditions for continuous-time T-S fuzzy systems, Journal of the Franklin Institute 353 (10) (2016) 2218-2236.

[9] R. Marquez, T. Guerra, M. Bernal, K. A., A non-quadratic lyapunov functional for $H_{\infty}$ control of nonlinear systems via takagi-sugeno models, Journal of the Franklin Institute 353 (4) (2016) 781-796.

[10] T. Gonzalez, A. Sala, M. Bernal, R. Robles, Invariant sets of nonlinear models via piecewise affine Takagi-Sugeno model, in: Proceedings of the 2015 IEEE International Conference on Fuzzy Systems, Istanbul, Turkey, 2015. 
[11] M. Johansson, A. Rantzer, K. Arzen, Piecewise quadratic stability of fuzzy systems, IEEE Transactions on Fuzzy Systems 7 (6) (1999) 713-722.

[12] C. Arino, E. Perez, A. Sala, B. F., Polytopic invariant and contractive sets for closed-loop discrete fuzzy systems, Journal of the Franklin Institute 351 (7) (2014) 3559-3576.

[13] H. Guo, J. Qiu, H. Tian, G. H., Fault detection of discrete-time T-S fuzzy affine systems based on piecewise lyapunov functions, Journal of the Franklin Institute 351 (7) (2014) 3633-3650.

[14] Y. Chen, M. Tanaka, K. Tanaka, H. Wang, Stability analysis and region-ofattraction estimation using piecewise polynomial lyapunov functions: Polynomial fuzzy model approach, IEEE Transactions on Fuzzy Systems 23 (4) (2015) 1314-1322.

[15] T. Hu, F. Blanchini, Non-conservative matrix inequality conditions for stability/stabilizability of linear differential inclusions, Automatica 46 (1) (2010) 190-196.

[16] J. Qiu, G. Feng, H. Gao, Static-output-feedback $H_{\infty}$ control of continuoustime T-S fuzzy affine systems via piecewise Lyapunov functions, IEEE Transactions on Fuzzy Systems 21 (2) (2013) 245-261.

[17] H. Schulte, H. Hahn, Fuzzy state feedback gain scheduling control of servopneumatic actuators, Control Engineering Practice 12 (2004) 639-650.

[18] F. Cuesta, A. Ollero, Fuzzy control of reactive navigation withstability analysis based on conicity and Lyapunov theory, Control Engineering Practice 12 (5) (2004) 625-638.

[19] H. Khalil, Nonlinear Systems, 3rd Edition, Prentice Hall, New Jersey, USA, 2002.

[20] T. Gonzalez, M. Bernal, Progressively better estimates of the domain of attraction for nonlinear systems via piecewise Takagi-Sugeno models: Stability and stabilization issues, Fuzzy Sets and Systems 297 (2016) 73-95. 
[21] J. Pitarch, A. Sala, C. Ariño, F. Bedate, Estimación del dominio de atracción de sistemas no lineales mediante modelos borrosos polinomiales (in spanish), Revista Iberoamericana de Automática e Informática Industrial 9 (2) (2012) 152-161.

[22] J. Pitarch, A. Sala, C. Ariño, Closed-form estimates of the domain of attraction for nonlinear systems via fuzzy-polynomial models, IEEE Transactions on Cybernetics 44 (4) (2014) 526-538.

[23] T. Gonzalez, M. Bernal, R. Marquez, Stability analysis of nonlinear models via exact piecewise Takagi-Sugeno models, in: Proceedings of the 19th IFAC World Congress, Cape Town, South Africa, 2014, pp. 1-6.

[24] S. Hedlund, M. Johansson, PWLTLOOL: A Matlab toolbox for analysis of Piecewise Linear Systems (1999).

[25] Z. Jarvis-Wloszek, R. Feeley, W. Tan, K. Sun, A. Packard, Control applications of sum of squares programming, in: Positive polynomials in control, Vol. 312 of Lecture Notes in Control and Information Science, Springer, 2005, pp. 3-22.

[26] U. Jönsson, A lecture on the S-procedure, in: http://people.kth.se/ $\sim$ uj/5B5746/Lecture.ps, Royal Institute of Technology, Stockholm, Sweden, 2001.

[27] H. Coexeter, Regular Polytopes, 3rd Edition, Methuen, Dover Publications, Toronto,Canada, 1973.

[28] K. Tanaka, H. Wang, Fuzzy Control Systems Design and Analysis. A linear matrix inequality approach, John Wiley and Sons, New York, 2001.

[29] J. Slotine, W. Li, Appplied Nonlinear Control, Prentice Hall, Englewood Cliffs, USA, 1991. 